3

4 Williams $\mathrm{N}^{1,2}$, Toselli $\mathrm{B}^{3}$, Siebenhühner $\mathrm{F}^{4}$, Palva $\mathrm{S}^{4,5}$, Arnulfo $\mathrm{G}^{3}$, Kaski $\mathrm{S}^{1,6 *}$, 5 Palva $\mathrm{JM}^{2,4,5 *}$

6

7

8

9

\section{Biophysical network models of phase-synchronization in MEG resting-state}

\section{Affiliations:}

1. Helsinki Institute of Information Technology, Department of Computer Science, Aalto University, Finland

2. Department of Neuroscience \& Biomedical Engineering, Aalto University, Finland

3. Department of Informatics, Bioengineering, Robotics \& Systems Engineering, University of Genoa, Italy

4. Neuroscience Center, Helsinki Institute of Life Science, University of Helsinki, Finland

5. Centre for Cognitive Neuroimaging, Institute of Neuroscience \& Psychology, University of Glasgow, United Kingdom

6. Department of Computer Science, University of Manchester, United Kingdom

* Equally contributing authors

Corresponding author:

Dr. Nitin Williams

Helsinki Institute of Information Technology

Department of Computer Science

Aalto University

Konemiehentie 2

02150, Espoo

6 Helsinki

Tel: +358 (0)449195512

38 Email: nitin.williams@aalto.fi 
39

40

\section{Abstract}

Magnetoencephalography (MEG) is used extensively to study functional connectivity (FC) networks of phase-synchronization, but the relationship of these networks to their biophysical substrates is poorly understood. Biophysical Network Models (BNMs) have been used to produce networks corresponding to MEG-derived networks of phase-synchronization, but the roles of inter-regional conduction delays, the structural connectome and dynamics of model of individual brain regions, in obtaining this correspondence remain unknown. In this study, we investigated the roles of conduction delays, the structural connectome, and dynamics of models of individual regions, in obtaining a correspondence between model-generated and MEGderived networks between left-hemispheric regions. To do this, we compared three BNMs, respectively comprising Wilson-Cowan oscillators interacting with diffusion Magnetic Resonance Imaging (MRI)-based patterns of structural connections through zero delays, constant delays and distance-dependent delays respectively. For the BNM whose networks corresponded most closely to the MEG-derived network, we used comparisons against null models to identify specific features of each model component, e.g. the pattern of connections in the structure connectome, that contributed to the observed correspondence. The WilsonCowan zero delays model produced networks with a closer correspondence to the MEGderived network than those produced by the constant delays model, and the same as those produced by the distance-dependent delays model. Hence, there is no evidence that including conduction delays improves the correspondence between model-generated and MEG-derived networks. Given this, we chose the Wilson-Cowan zero delays model for further investigation. Comparing the Wilson-Cowan zero delays model against null models revealed that both the pattern of structural connections and Wilson-Cowan oscillatory dynamics contribute to the correspondence between model-generated and MEG-derived networks. Our investigations yield insight into the roles of conduction delays, the structural connectome and dynamics of models of individual brain regions, in obtaining a correspondence between model-generated and MEG-derived networks. These findings result in a parsimonious BNM that produces networks corresponding closely to MEG-derived networks of phase-synchronization.

Keywords: Biophysical Network Models; Wilson-Cowan oscillators; Phase-synchronization; Magnetoencephalography; MEG resting-state; MEG functional networks 
73

74

75

76

77

78

79

80

81

82

83

84

85

86

87

88

89

90

91

92

93

94

95

96

97

98

99

100

101

102

01

Highlights

- Simple biophysical model produces close match $(\rho=0.49)$ between model and MEG networks

- No evidence for conduction delays improving match between model and MEG networks

- Pattern of structural connections contributes to match between model and MEG networks

- Wilson-Cowan oscillatory dynamics contribute to match between model and MEG networks

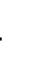

85




\section{Introduction}

104 Functional Connectivity (FC) networks underpin the execution of cognitive tasks (Cole et al. 105 (2013), Cohen \& D’Esposito (2016)) and are also observed during resting-state (Beckmann et 106 al. (2005), Damoiseaux et al. (2006)). FC networks are sets of interacting brain regions, 107 wherein the interactions are reflected by correlated activities of the brain regions. Key forms 108 of correlation observed in Electroencephalography (EEG) and Magnetoencephalography 109 (MEG) resting-state or task data, are the pairwise correlations between the oscillation 110 amplitudes of a set of brain regions (Hipp et al. (2012), Colclough et al. (2015), Brookes et al. 111 (2012)), or the pairwise synchronization between oscillation phases of a set of brain regions 112 (Palva et al. (2005), Palva et al. (2010), Palva \& Palva (2012), Zhigalov et al. (2015), 113 Siebenhühner et al. (2020), Marzetti et al. (2019)). FC networks of amplitude correlation or 114 phase-synchronization are widely studied with EEG/MEG, but the structure-function mapping 115 between biophysical substrates and FC networks is poorly understood. We refer to biophysical substrates as systems-level components, e.g., the structural connectome, dynamics of brain regions or inter-regional conduction delays, which underpin observed FC networks.

Biophysical Network Models (BNMs) (Woolrich \& Stephan (2013)) have been used to relate MEG-derived networks of amplitude correlation to their biophysical substrates. BNMs comprise models of individual brain regions, linked by biologically informed structural connectivity. BNMs of Kuramoto oscillators linked by diffusion Magnetic Resonance Imaging (dMRI)-based structural connections with realistic conduction delays, produced FC networks of amplitude correlation corresponding moderately to those observed in MEG resting-state (Cabral et al. (2014)) ( $\rho=0.41$, where $\rho$ is Pearson Correlation). Similarly, BNMs of spiking neuron populations linked by dMRI-based structural connections with realistic delays also produced FC networks of amplitude correlation corresponding moderately to those observed in MEG-derived networks of amplitude correlation $(\rho=0.4)$ (Nakagawa et al. (2014)).

FC networks of phase-synchronization have been suggested to be relevant to information processing in the brain, coordinating communication across regions via regulation of spiketime relationships (Fries (2005), Singer (1999), Palva \& Palva (2012)) and supporting functional integration (Palva \& Palva, (2012), Siegel et al. (2012), Deco et al. (2015)). In fact, particular phase-synchronization networks are known to be recruited for specific cognitive 
135

136

137

138

139

140

141

142

143

144

145

146

147

148

149

150

151

152

153

154

155

156

157

158

159

160

161

162

163

164

165

166

167

168

tasks, including working memory (Kitzbichler et al. (2011), Palva et al. (2010)), visual attention (Lobier et al. (2018)) and sensorimotor processing tasks (Hirvonen et al. (2018)).

Just as for MEG-derived networks of amplitude correlation, BNMs have been employed to relate EEG- or MEG-derived networks of phase-synchronization to their biophysical substrates. BNMs of Kuramoto oscillators linked by dMRI-based structural connections with conduction delays, produced networks of phase-synchronization corresponding to those observed in EEG resting-state (Finger et al. (2016)). However, this model produced networks with only weak correspondence to EEG-derived networks, when the networks were estimated with measures insensitive to EEG volume conduction $(\rho=0.17$ for weighted Phase Lag Index (wPLI)). BNMs of Wilson-Cowan oscillators with inhibitory synaptic plasticity (ISP), linked by dMRI-based structural connections with conduction delays, have been used to produce networks of phase-synchronization corresponding to those observed in MEG resting-state (Abeysuriya et al. (2018)). While this BNM produced networks with a moderate correlation to MEG-derived networks ( $\rho=0.43$ for Phase Locking Value (PLV), $\rho=0.28$ for Phase Lag Index (PLI)) (Abeysuriya et al. (2018)), the strengths of model-generated phasesynchronization ( $~ 0$ - 0.6 for PLV and $\sim 0$ - 1 for PLI) were an order of magnitude higher than those observed in MEG-derived networks ( 0.01 - 0.06 for both PLV and PLI). Further, while the correspondence between the model-generated and MEG-derived networks was assessed, the roles of conduction delays, the structural connectome and the dynamics of models of individual brain regions in producing this correspondence was not investigated.

In this study, we investigated the roles of conduction delays, the structural connectome and the dynamics of models of individual regions, in producing networks corresponding to observed MEG-derived networks of phase-synchronization between left-hemispheric regions. To do this, we first compared three hypotheses, respectively postulating model-generated networks of phase-synchronization corresponding to MEG-derived networks, are produced by the dynamics of Wilson-Cowan oscillators linked by dMRI-based patterns of structural connections, via zero, constant and distance-dependent conduction delays. We expressed each of these hypotheses as a BNM. For the BNM producing networks corresponding most closely to the observed MEG-derived networks, we used comparisons against null models to determine specific features of each model component, for e.g., pattern of connections in the structural connectome, that contribute to the correspondence between the model-generated and MEGderived networks. Together, these investigations yield insight into the precise roles of inter- 
regional conduction delays, the structural connectome and the dynamics of models of

170 individual brain regions, in producing networks corresponding to observed MEG-derived networks of phase-synchronization.

\section{2. Materials \& Methods}

173 In this study, we investigated the roles of conduction delays, the structural connectome and the

174 dynamics of models of individual brain regions, in producing model-generated networks 175 corresponding to the observed MEG-derived networks of phase-synchronization. To do this, 176 we first compared the correspondence between model-generated and MEG-derived networks 177 of three BNMs, respectively including zero, constant and distance-dependent conduction 178 delays. Figure 1 illustrates the pipeline to estimate and compare the model-generated and MEG179 derived networks. For the BNM producing networks corresponding most closely to the MEG180 derived networks, we used null models to determine specific features of each model component 181 that contributes to the correspondence between model-generated and MEG-derived networks. 182 For example, we compared the correspondence between model-generated networks from the original model and the MEG-derived networks, against the correspondence between modelgenerated networks from null models comprising degree-preserved randomised versions of the structural connectome, and the MEG-derived networks. Such a comparison enabled inferences on the contribution of the pattern of structural connections to the correspondence between model-generated and MEG-derived networks, over and above the number of connections, i.e. degree, to each brain region. 

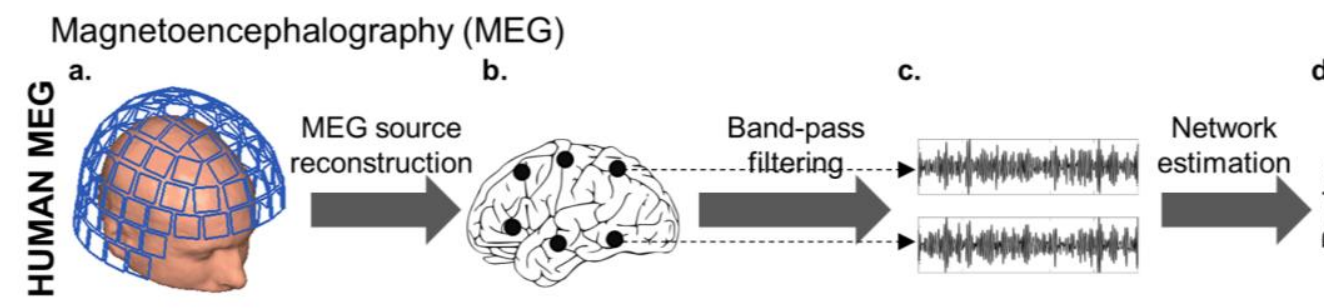

d.

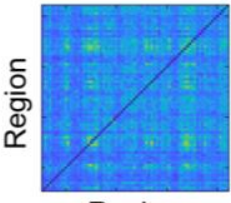

Region

Biophysical Network Model (BNM) Link between oscillators

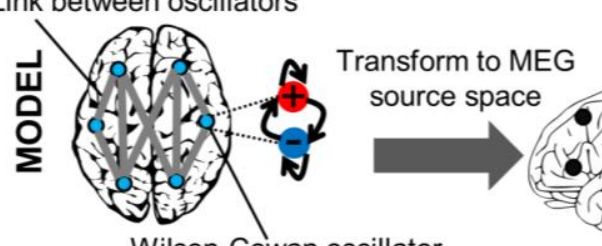

Wilson-Cowan oscillator

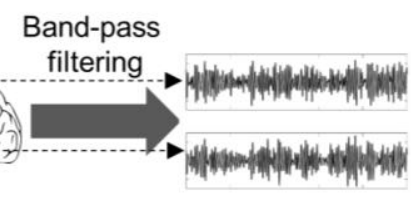

h.
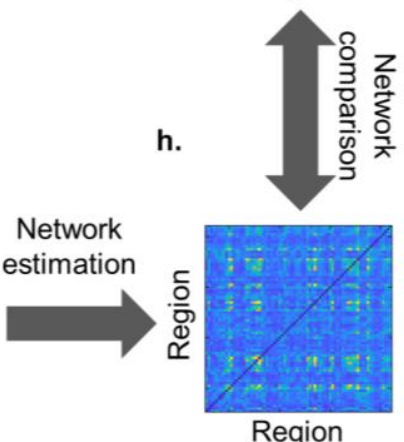

Figure 1. Pipeline to generate and compare model-generated and MEG-derived networks

of phase-synchronization. a. MEG device with sensors detecting weak magnetic fields arising from post-synaptic potentials in the human brain. b. Source-reconstructed data projected from MEG sensors to brain regions. c. Band-pass filtered source-reconstructed MEG data. d. Matrix of strength of phase-synchronization between source-reconstructed MEG data from every pair of brain regions. e. Biophysical Network Model (BNM) comprising Wilson-Cowan oscillators linked by biologically informed structural connections. f. Simulated data transformed to MEG source space by projecting dynamics to MEG sensors, and projecting sensor-level simulated data back to brain regions. g. Band-pass filtered source-reconstructed simulated MEG data. h. Matrix of strength of phase-synchronization between source-reconstructed simulated MEG data from every pair of brain regions. MEG device panel from Pfeiffer et al. (2018).

\subsection{Models}

204 We implemented three models, each representing specific hypotheses on the interaction between dynamics of models of brain regions, structural connectome and conduction delays, in producing networks corresponding to MEG-derived networks of phase-synchronization.

Wilson-Cowan zero delays model: Wilson-Cowan oscillatory dynamics interact with the pattern of connections in the structural connectome without conduction delays, to produce 
We implemented this model as a set of Wilson-Cowan oscillators linked by biologically informed structural connectivity (Wilson \& Cowan (1972), Kilpatrick (2013), Cowan (2016)).

214 The dynamics of each oscillator arise from the interaction between excitatory and inhibitory neuronal populations, i.e. the PING model (Traub et al. (1997)), and are also influenced by external inputs and the dynamics of linked oscillators. For oscillator $i$ :

$$
\tau_{e} \frac{d U_{e}}{d t}=-U_{e}(t)+F\left(w_{e e} U_{e}(t)-w_{e i} U_{i}(t)-b_{e}+J_{e}+k \sum_{j=1}^{N} K(i, j) U_{e}(t)\right)
$$

$$
\tau_{i} \frac{d U_{i}}{d t}=-U_{i}(t)+F\left(w_{i e} U_{e}(t)-w_{i i} U_{i}(t)-b_{i}+J_{i}\right)
$$
$t$ of the excitatory and inhibitory populations respectively, $w_{e e}$ and $w_{i i}$ are the excitatoryexcitatory and inhibitory-inhibitory connection weights respectively, $w_{i e}$ and $w_{e i}$ are the excitatory-inhibitory and inhibitory-excitatory connection weights, $b_{e}$ and $b_{i}$ are threshold constants for excitatory and inhibitory populations, $J_{e}$ and $J_{i}$ are injection currents to excitatory and inhibitory populations, $\tau_{e}$ and $\tau_{i}$ are the time constants of the excitatory and inhibitory populations, and $k$ is a scalar multiplier over the coupling matrix $K$, which specifies links between $N$ oscillators. For this model, we postulated that non-zero conduction delays are not relevant to producing the networks of phase-synchronization observed in MEG resting-state.

Parameter values: We set the parameters of each Wilson-Cowan oscillator to the same values (Table 1) since we assumed the intrinsic properties of the brain regions are identical. The choice of values for connection weights within $\left(w_{e e}, w_{i i}\right)$ and between $\left(w_{i e}, w_{e i}\right)$ excitatory and inhibitory populations, and the strengths of external currents $\left(J_{e}, J_{i}\right)$ are consistent with canonical neurophysiological findings of 1.) strong excitatory-excitatory connections (Jansen \& Rit (1995), Douglas et al. (1989), Binzegger et al. (2004)) 2.) excitatory-inhibitory and inhibitory-excitatory connections being weaker than excitatory-excitatory connections (Jansen \& Rit (1995), Binzegger et al. (2004)) 3.) weak inhibitory-inhibitory connections (Douglas et al. (1989), Binzegger et al. (2004)) and 4.) the strength of injection currents, e.g. from thalamus, being significantly lower than strength of excitatory-excitatory connections (Douglas et al. (1989), Binzegger et al. (2004)). The threshold constants of excitatory $\left(b_{e}\right)$ and inhibitory populations $\left(b_{i}\right)$ are difficult to determine from the neurophysiology literature, but the values were chosen to produce oscillatory phenomena (Wilson \& Cowan (1972), Singh et al. (2016), 
243 Sreenivasan et al. (2017)). We chose the excitatory $\left(\tau_{e}\right)$ and inhibitory time constants $\left(\tau_{i}\right)$ to 244 produce dynamics around $10 \mathrm{~Hz}$, i.e. the peak frequency of oscillatory power in the power 245 spectrum of MEG resting-state (Nakagawa et al. (2014)). We tuned the parameter $k$, i.e. the 246 scalar multiplier over the coupling matrix, $K$ (see Section 2.6).

\begin{tabular}{|l|l|}
\hline Parameters & Values \\
\hline$w_{e e}, w_{i i}, w_{i e}, w_{e i}$ & $16,3,12,15$ \\
\hline$b_{e}, b_{i}$ & $4,3.7$ \\
\hline$J_{e}, J_{i}$ & 2,0 \\
\hline$\tau_{e}, \tau_{i}$ & $23.7,23.7$ \\
\hline
\end{tabular}

Table 1. Parameter values of individual Wilson-Cowan oscillators

Wilson-Cowan constant delays model: Wilson-Cowan oscillatory dynamics interact with the pattern of connections in the structural connectome through constant non-zero conduction delays, to produce networks of phase-synchronization corresponding to those observed in MEG resting-state.

We implemented this model as a set of Wilson-Cowan oscillators linked by biologically informed structural connectivity with constant, non-zero delays. The dynamics of individual oscillators were influenced by inter-regional interactions of constant delay, in contrast to zero delays assumed by the Wilson-Cowan zero delays model. For oscillator $i$ :

$$
\tau_{e} \frac{d U_{e}}{d t}=-U_{e}(t)+F\left(w_{e e} U_{e}(t)-w_{e i} U_{i}(t)-b_{e}+J_{e}+k \sum_{j=1}^{N} K(i, j) U_{e}(t-T)\right)
$$

$$
\tau_{i} \frac{d U_{i}}{d t}=-U_{i}(t)+F\left(w_{i e} U_{e}(t)-w_{i i} U_{i}(t)-b_{i}+J_{i}\right)
$$

where $T$ specifies the constant delay in milliseconds, and all other terms bear the same definitions as the Wilson-Cowan zero delays model. For this model, we postulated that the increase in conduction delay due to longer distance between regions is counteracted by the decrease in conduction delay due to higher conduction velocity between regions. This 
assumption is supported by recent modelling work (Noori et al. (2020)) reporting highly similar conduction delays across region-pairs due to increased conduction velocity produced by activity-dependent myelination counteracting longer delays between distant brain regions.

Parameters values: We set the parameters of the Wilson-Cowan oscillators to the values in Table 1, and tuned the parameters $k$ and constant delay $T$ (see Section 2.6).

273

274

Wilson-Cowan distance-dependent delays model: Wilson-Cowan oscillatory dynamics interact with the pattern of connections in the structural connectome through distance-dependent conduction delays, to produce networks of phase-synchronization corresponding to those observed in MEG resting-state.

We implemented the Wilson-Cowan distance-dependent delays model as comprising a set of Wilson-Cowan oscillators linked by biologically informed structural connectivity, with conduction delays proportional to distance between respective brain regions. The dynamics of individual oscillators are influenced by inter-regional interactions of variable delay, in contrast to zero and constant delays assumed by the Wilson-Cowan zero delays and constant delays models respectively. For oscillator $i$ :

$$
\tau_{e} \frac{d U_{e}}{d t}=-U_{e}(t)+F\left(w_{e e} U_{e}(t)-w_{e i} U_{i}(t)-b_{e}+J_{e}+k \sum_{j=1}^{N} K(i, j) U_{e}(t-T(i, j))\right)
$$

where $T(i, j)$ specifies the distance-dependent delay in metres/second, and all other terms bear

$$
\tau_{i} \frac{d U_{i}}{d t}=-U_{i}(t)+F\left(w_{i e} U_{e}(t)-w_{i i} U_{i}(t)-b_{i}+J_{i}\right)
$$
the same definitions as the model describing the Wilson-Cowan zero delays model. For this model, we specified conduction delays as Euclidean distance between regions divided by a conduction velocity $v$ (Cabral et al. (2014), Nakagawa et al. (2014), Hadida et al. (2018)).

Rather than Euclidean distance, an alternative method to estimate distances between brain regions is the average length of the streamlines between regions, as calculated by the tractography method used. However, we found the average estimated streamline lengths of $\sim 20 \%$ of structural connections to be lower than the corresponding Euclidean distances, which represent the minimum length between regions. Since this might be due to known biases in 
streamline termination with tractography methods (Smith et al. (2013)), we chose Euclidean distance to estimate distances between brain regions.

Parameters values: We set the parameters of the Wilson-Cowan oscillators to the values in

Table 1 , and tuned the parameters $k$ and the conduction velocity $v$ (see Section 2.6).

\subsection{Model comparison}

305

We compared the Wilson-Cowan zero delays, constant delays and distance-dependent delays models by the correspondence between their respective networks of phase-synchronization and the observed MEG-derived network of phase-synchronization. We ran 20 simulations of each model to obtain its dynamics at different starting conditions. After each run, we compared the model-generated network of phase-synchronization to a held-out MEG-derived network of phase-synchronization, i.e. an MEG-derived network not used to estimate model parameters (see Section 2.5). We quantified correspondence between model-generated and MEG-derived networks, i.e. model performance, by the RMSE (Root Mean Square Error) and Pearson Correlation between the upper-triangular elements of the model-generated and MEG-derived adjacency matrices, low RMSE and high Correlation reflecting a close correspondence. We used independent samples $t$-tests of the respective RMSE and Correlation values to compare model performances, with two-tailed $p<0.001$ considered statistically significant. Further, we used Cohen's $d$ (Cohen (1988)) to measure effect sizes, with $d \geq 1$ considered a strong effect.

\subsection{Obtaining model-generated network of phase-synchronization}

Below, we describe the details of the different model components, model simulations, and the procedure to generate the network of phase-synchronization from the model dynamics.

\subsubsection{Model elements}

324 The model comprised an ensemble of 74 Wilson-Cowan oscillators linked by biologically informed structural connectivity. We used the left-hemispheric regions of the Destrieux brain atlas (Destrieux et al. (2010)) to specify the number and location of the oscillators. We specified the links between oscillators by the binary matrix of the strongest 10 percentile structural connections between left-hemispheric regions of the Destrieux atlas. We derived this binary matrix from a group-averaged $(\mathrm{N}=57)$ weighted matrix of the number of streamlines between brain regions, estimated by constrained spherical deconvolution (Smith et al. (2013)) and probabilistic tractography (Smith et al. (2012)) on pre-processed DWI images from the Human 
Connectome Project (van Essen et al. (2013)). We divided each element of the binary matrix by the sum of elements in its row, to ensure similar strengths of inputs to each of the 74 regions (Hlinka \& Coombes (2012), Forrester et al. (2020)).

\subsubsection{Model simulations}

337 We used the Brain Dynamics Toolbox (BDT) for model simulations (Heitmann et al. (2018)).

338 The Wilson-Cowan zero delays model is implemented in BDT, while we extended the BDT implementation of the zero delays model to implement the Wilson-Cowan constant delays and Wilson-Cowan distance-dependent delays models. We have made these implementations publicly available (Williams et al. (2021a)). We simulated the models for 630 seconds at 500 Hz with ODE45 (Bogacki \& Shampine (1996)) for the Wilson-Cowan zero delays model, and ODE23a (Shampine \& Thompson (2001)) for the constant delays and distance-dependent delays models. We limited local discretisation error by setting Absolute and Relative Tolerance of all solvers to $1 \times 10^{-6}$ and $1 \times 10^{-3}$ respectively. We set initial conditions of excitatory and inhibitory populations of the Wilson-Cowan oscillators by sampling a uniform random distribution between 0 and 1 . Notably, we used dynamics of only the excitatory populations for further processing since pyramidal neurons in excitatory populations are the dominant contributors to the measured MEG (Lopes da Silva (2013)). These model dynamics represented the mean firing rate of pyramidal neurons in excitatory populations.

\subsubsection{Transforming simulated data to MEG source-space}

We transformed simulated data to MEG source-space by forward-projecting the simulated data to MEG sensors and then inverse-projecting the simulated sensor-level data, with subjectspecific forward and inverse operators respectively (Korhonen et al. (2014)). We used the same forward and inverse operators estimated to source-reconstruct the sensor-level MEG restingstate data (see Section 2.4.2). This procedure yielded subject-specific source-reconstructed simulated MEG datasets, of activity from 74 regions for 630 seconds.

\subsubsection{Band-pass filtering}

We band-pass filtered the model dynamics in the alpha frequency band $(8-12 \mathrm{~Hz})$ since it is the dominant source of oscillatory power in MEG resting-state (Nakagawa et al. (2014)). We performed the filtering with Morlet wavelets of peak frequency $=9.83 \mathrm{~Hz}$, width parameter $=$ 5, yielding subject-specific narrowband datasets, from 74 regions for 630 seconds. 


\subsubsection{Estimating network of phase-synchronization}

For each subject-specific dataset, we used weighted Phase Lag Index (wPLI) (Vinck et al. (2011)) to estimate strength of phase-synchronization between each pair of brain regions.

$$
w P L I=\frac{\mid E(|\operatorname{Imag}(X)| \operatorname{sign}(\operatorname{Imag}(X)) \mid}{E(|\operatorname{Imag}(X)|)}
$$

where $X$ is the cross-spectrum between a pair of signals and $\operatorname{Imag}(X)$ is its imaginary component. Crucially, wPLI is insensitive to the confounding influence of MEG field spread

374 (Vinck et al. (2011), Palva et al. (2018)). We removed the effect of transient dynamics on the phase-synchronization estimates by discarding the first 30 seconds of each dataset. The phasesynchronization estimation produced $74 \times 74$ subject-specific matrices, which we averaged to obtain the model-generated network of phase-synchronization.

\subsection{Obtaining MEG-derived network of phase-synchronization}

Below, we describe the details of acquiring, preprocessing, source-reconstructing sensor-level MEG resting-state data and estimating the MEG-derived network of phase-synchronization.

\subsubsection{Data acquisition \& pre-processing}

384 We recorded MEG resting-state data from 46 healthy subjects (19 female, age $30.7 \pm 9.5$ years) for 600 seconds at a sampling rate of $1000 \mathrm{~Hz}$. During the recordings, we instructed subjects to focus on a cross on the screen in front of them during the recordings. We performed the recordings at Meilahti hospital, Helsinki with a 306-channel MEG system (204 planar gradiometers and 102 magnetometers, MEGIN Oy). For each subject, we collected T1weighted anatomical MRI scans at a resolution of $1 \times 1 \times 1 \mathrm{~mm}$ with a $1.5 \mathrm{~T}$ MRI scanner the study, and we performed the study according to the Declaration of Helsinki. We obtained written informed consent from each participant. We used MaxFilter (Taulu \& Hari (2009)) to suppress extra-cranial noise and co-localise the sensor-space recordings, and Independent Component Analysis (ICA) (Oostenveld et al. (2011)), to exclude components identified as ocular or cardiac artefacts. 


\subsubsection{Estimating MEG-derived network of phase-synchronization}

We transformed preprocessed, sensor-level MEG data time series to brain regions, then bandpass filtered these data and estimated networks of phase-synchronization. We used MNE software to generate subject-specific inverse operators (Hämäläinen \& Ilmoniemi (1994)) to transform sensor-level data to the level of cortical parcels or brain regions (Palva et al. (2010), Zhigalov et al. (2017), Lobier et al. (2018), Hirvonen et al. (2018)). We applied fidelity weighting (Korhonen et al. (2014)) on the inverse operators to reduce the influence of MEG field spread. We also used MNE to generate subject-specific forward operators, including subject-specific head conductivity models, cortically constrained source models with 50007500 sources per hemisphere, and to co-localise MEG to MRI. We used FreeSurfer (http://surfer.nmr.mgh.harvard.edu/) for volumetric segmentation of MRI data, surface reconstruction, flattening, cortical parcellation and neuroanatomical labelling of each source to a region in the Destrieux brain atlas. For each subject, we averaged the time series of sources within a region to obtain the activity time-course for that region. We then downsampled each subject-specific dataset to $500 \mathrm{~Hz}$ and retained the time courses of the 74 left-hemispheric regions for further processing. We used an identical procedure as applied to the simulated data, to perform band-pass filtering (see Section 2.3.4) and to estimate subject-specific $74 \times 74$ MEG-derived matrices of phase-synchronization (see Section 2.3.5).

\subsection{Splitting MEG-derived networks into training and testing datasets}

We generated two subsets of the original 46 subject-specific phase-synchronization matrices, to estimate free model parameters and to evaluate model performance respectively. The first subset or training dataset contained 23 unique, randomly selected subject-specific matrices. We averaged the matrices to obtain a group-level matrix, which we used as the target MEG-derived network to estimate model parameters. The held-out or testing dataset contained the 23 subjectspecific matrices not used in the first subset. We averaged these matrices to obtain a grouplevel matrix, which we used as the target MEG-derived network to determine correspondence between model-generated and MEG-derived networks.

\subsection{Determining optimal values of model parameters}

We selected optimal model parameter values by performing a grid search for a range of parameter values. We quantified correspondence between model-generated and MEG-derived networks, i.e., model performance, as RMSE and Pearson Correlation between the uppertriangular elements of the model-generated and the training set MEG-derived matrices of 
phase-synchronization (see Section 2.5). We chose optimal parameter values as those simultaneously yielding low RMSE and high Correlation. For the zero delays, constant delays and distance-dependent delays models, we used the parameter range of $k=0.5$ to 5 , in intervals of 0.5 , for the scalar multiplier over the structural connectome. For the constant delays model, we had a delay parameter $T$, with range from $2 \mathrm{~ms}$ to $20 \mathrm{~ms}$, in intervals of $2 \mathrm{~ms}$. For the distance-dependent delays model, we had a conduction velocity parameter $v$, with range from $3 \mathrm{~m} / \mathrm{s}$ to $30 \mathrm{~m} / \mathrm{s}$, in intervals of $3 \mathrm{~m} / \mathrm{s}$. We ran simulations for 315 seconds for each parameter combination and discarded the first 30 seconds to remove transient model dynamics, before estimating the model-generated network of phase-synchronization.

\subsection{Evaluating statistical significance of model performance}

444 We evaluated statistical significance of model performance by comparing against permutationbased null distributions of the performance measures. We quantified model performance by RMSE and Correlation between upper-triangular elements of the mean model-generated matrix of phase-synchronization and the held-out testing set MEG-derived matrix of phasesynchronization (see Section 2.5). We estimated the mean model-generated matrix of phasesynchronization by averaging the 20 model-generated matrices from different starting conditions. We determined statistical significance of the RMSE and Correlation values by zscoring them against 100 corresponding values estimated after randomly permuting, without replacement, the mean model-generated matrix. We tested the hypotheses that RMSE is lower than chance and that Correlation is higher than chance by calculating the one-tailed $p$-value of the $z$-scores, with $p<0.001$ considered statistically significant.

\subsection{Sensitivity of model performance to choice of model parameter values}

We assessed the sensitivity of the model's performance to small changes in parameter values. To do this, we determined RMSE and Correlation values between the MEG-derived matrix and model-generated matrices generated from simulating jittered models. To introduce jitter, each non-zero parameter of the model was varied between $-10 \%$ and $10 \%$ of its original value, with $2 \%$ increments, while keeping all other parameters at their original values. Then, we $z$-scored the RMSE and Correlation values against the 20 RMSE and Correlation values from the original model. $z$ values higher than 3 were considered to reflect values of parameters at which the model performance was sensitive. 


\subsection{Determining features of each model component contributing to model performance}

For the model producing networks of phase-synchronization corresponding most closely to the MEG-derived networks of phase-synchronization, we determined specific features of each model component, e.g., pattern of connections in the structural connectome, contributing to the observed correspondence. To do this, we compared the correspondence between modelgenerated and MEG-derived networks obtained with the original model, against the correspondence obtained with 100 examples of a null model, each containing a randomised version of a specific model component, e.g., randomly connected structural connectome, while all other aspects of the model remained identical to the original model.

Each of the Wilson-Cowan zero delays, constant delays and distance-dependent delays models, postulate that the topological organisation of the structural connectome, i.e., its pattern of connections, contributes to the correspondence between model-generated and MEG-derived networks. To verify this, we created 100 examples of a null model containing degree-preserved but randomised versions (Maslow \& Sneppen (2002)) of the original binary structural connectome, while all other aspects were identical to the original model. For each example of this null model, the randomised structural connectome had the same number of connections to each brain region as the original structural connectome, but the pattern of connections between regions was different to the original structural connectome. By comparing the correspondence between model-generated and MEG-derived networks of the original model against the correspondences obtained with the null models, we tested the hypothesis that the pattern of structural connections contributed to the observed correspondence, over and above the number of connections, i.e., degree, to each brain region.

491 Each of the Wilson-Cowan zero delays, constant delays and distance-dependent delays models postulate that the Wilson-Cowan oscillatory dynamics contribute to the correspondence between model-generated and MEG-derived networks. To verify this, we created 100 examples of a null model where the dynamics of each brain region were described by a uniform random distribution whose mean $(0.2)$ and range $(0.07$ to 0.36$)$ were matched to those of original Wilson-Cowan dynamics, while the original structural connectome specified the interaction 


$$
\frac{d U}{d t}=-U(t)+F\left(\operatorname{rand}(t)+k \sum_{j=1}^{N} K(i, j) U(t-T(i, j))\right)
$$

499

500

501

502

503

504

505

506

507

508

509

510

511

512

513

514

515

516

517

518

519

520

521

522

523

524

525

526

527

528

529

where $F(v)=\frac{1}{1+e^{-v}}$ is a sigmoid function, $U(t)$ is the mean firing rate, $\operatorname{rand}(t)$ is a sample from a uniform random distribution, $k$ is a scalar multiplier over the coupling matrix $K$, which specifies links between $N$ oscillators and $T(i, j)$ is a delay matrix containing zeros for all elements, for the Wilson-Cowan zero delays model, a constant non-zero delay for all elements, for the Wilson-Cowan constant delays model, and connection-specific delays for each element, for the Wilson-Cowan distance-dependent delays model. We estimated the parameter $k$ using the same procedure as for the original model (see Section 2.6). We set Relative Tolerance of the simulation to 0.1 , to ensure completion in a reasonable time. By comparing the correspondence between model-generated and MEG-derived networks of the original model against the correspondences obtained with the null models, we tested the hypothesis that the Wilson-Cowan oscillatory dynamics contributed to the correspondence between modelgenerated and MEG-derived networks of phase-synchronization, over and above random dynamics with same mean and range as the Wilson-Cowan oscillatory dynamics.

The Wilson-Cowan distance-dependent delays model postulated that the connection-specific delays contributed to the correspondence between the model-generated and MEG-derived networks. To verify this, we created 100 examples of a null model by randomly permuting delays (without replacement) from the original model, while all other aspects, including the structural connectome and Wilson-Cowan oscillatory dynamics, were identical to the original model. By comparing the correspondence between model-generated and MEG-derived networks of the original model against the correspondences obtained with the null models, we tested the hypothesis that the connection-specific delays contributed to the observed correspondence, over and above merely the set of conduction delays used. Since the WilsonCowan zero delays and Wilson-Cowan constant delays models had the same delays for each connection, determining the contribution of connection-specific delays to the correspondence between model-generated and MEG-derived networks was not relevant.

Correspondence between the model-generated and MEG-derived networks, for both the original model and null models, were quantified by RMSE and Correlation between the uppertriangular elements of model-generated and the testing set MEG-derived matrices of phasesynchronization (see Section 2.5). To remove outliers from the null distribution, we excluded 
530

531

532

533

534

535

536

537

538

539

540

541

542

543

544

545

546

547

548

549

550

551

552

553

554

555

556

557

558

559

560

cases for which RMSE values lay more than 5SD from the mean. We then estimated the $z-$ score of the original model's RMSE and Correlation against RMSE and Correlation of the null models. We tested the hypotheses that mean RMSE for the original model is lower than RMSE for the null models and mean Correlation for the original model is higher than Correlation for the null models by calculating one-tailed p-values of the respective $z$-scores, with $p<0.001$ considered a statistically significant contribution.

\subsection{Comparing model performance against performance of Kuramoto oscillator model}

For the model producing networks of phase-synchronization corresponding most closely to those observed in MEG resting-state, we compared its observed correspondence against the correspondence between model-generated and MEG-derived networks of an equivalent Kuramoto oscillator model (Kuramoto (1984)). The Kuramoto oscillator model has been used to model oscillatory phenomena in systems neuroscience (Breakspear et al. (2010), Cabral et al. (2014)). The phase dynamics of individual regions were described by the classic Kuramoto model, where we used the same coupling matrix $K$ as in the Wilson-Cowan models, to specify links between regions. The sine of instantaneous phases was computed to convert the phase dynamics to time series of oscillatory activity. We set the scalar multiplier $k$ over the coupling matrix with the same training procedure used for the Wilson-Cowan zero delays model (Section 2.6), while we set the natural frequencies of the Kuramoto oscillators so as to produce oscillatory dynamics around $10 \mathrm{~Hz}$. To do this, we set the natural frequencies as independent samples from a Gaussian distribution of mean $=6.2 \times 10^{-2}$ and standard deviation $=6.2 \times$ $10^{-3}$. We simulated the model, then applied the same processing as to the Wilson-Cowan model dynamics (see Section 2.3) to furnish the Kuramoto model-generated matrix of phasesynchronization, which we then matched to the MEG-derived matrix with RMSE and Correlation. We used independent samples $t$-tests to compare RMSE and Correlation values of the chosen model against RMSE and Correlation values of the Kuramoto model, with twotailed $p<0.001$ considered statistically significant.

\section{Results}

\subsection{Determining optimal values of model parameters}

We estimated the optimal values of free parameters, for the Wilson-Cowan zero delays, constant delays and distance-dependent delays models. We identified the optimal parameter 
values as those that yielded model-generated matrices of phase-synchronization with the strongest correspondence to the training set MEG-derived matrices of phase-synchronization.

563 For each of the models, we compared model-generated and MEG-derived matrices of phase-

564 synchronization using RMSE and Pearson Correlation, choosing parameter values yielding low

565 RMSE and high Correlation (Figure 2). For the Wilson-Cowan zero delays model, the optimal 566 parameter value is $k=2$, yielding $\mathrm{RMSE}=0.04$ and Correlation $=0.45, k$ being the scalar 567 multiplier over the structural connectome. For the Wilson-Cowan constant delays model, the 568 optimal combination of parameter values is $k=2$ and $T=2 \mathrm{~ms}$, yielding RMSE $=0.04$ and 569 Correlation $=0.33$, with $T$ being the constant delay between brain regions. For the Wilson570 Cowan distance-dependent delays model, the optimal combination of parameter values is $k=$ $571 \quad 1.5$ and $v=12 \mathrm{~m} / \mathrm{s}$, yielding RMSE $=0.04$ and Correlation $=0.38$, with $v$ being the assumed 572 transmission velocity of neuronal activity across structural connections. We used these values 573 to run fresh simulations of the Wilson-Cowan zero delays, constant delays and distance574 dependent delays models, and determined correspondences of the respective model-generated 575 matrices of phase-synchronization to the testing set MEG-derived matrix of phase576 synchronization, which we estimated as the average of 23 held out subject-level matrices. 
a. Wilson-Cowan zero delays model

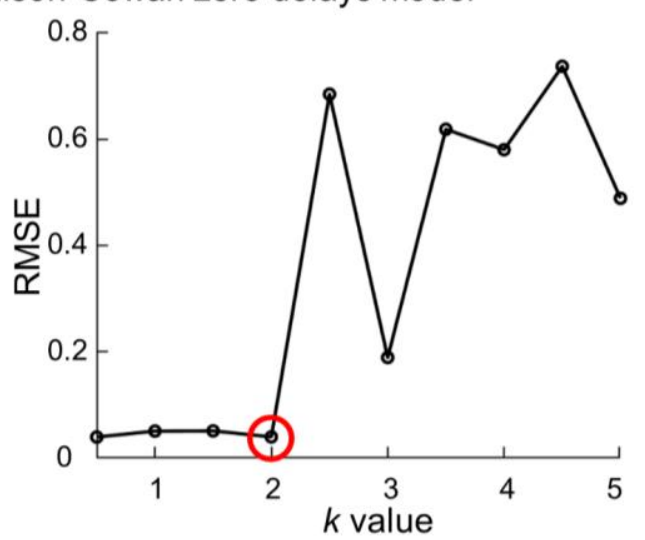

b. Wilson-Cowan constant delays model

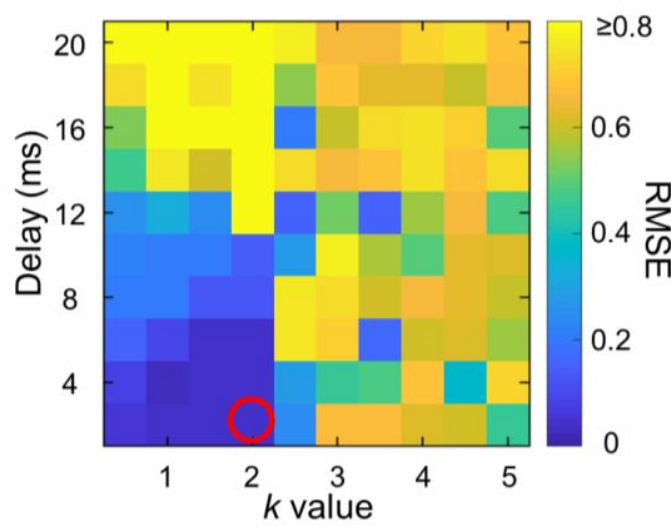

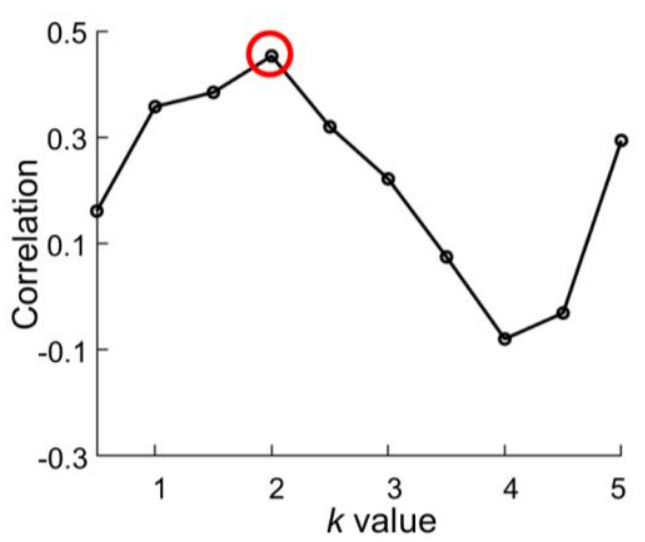

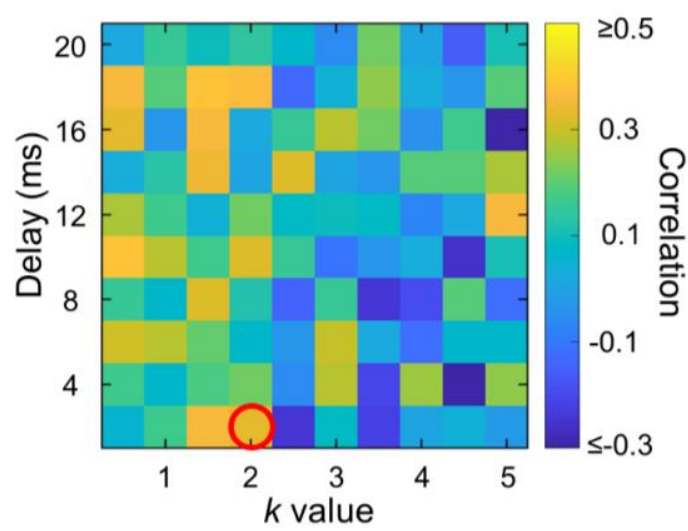

c. Wilson-Cowan distance-dependent delays model
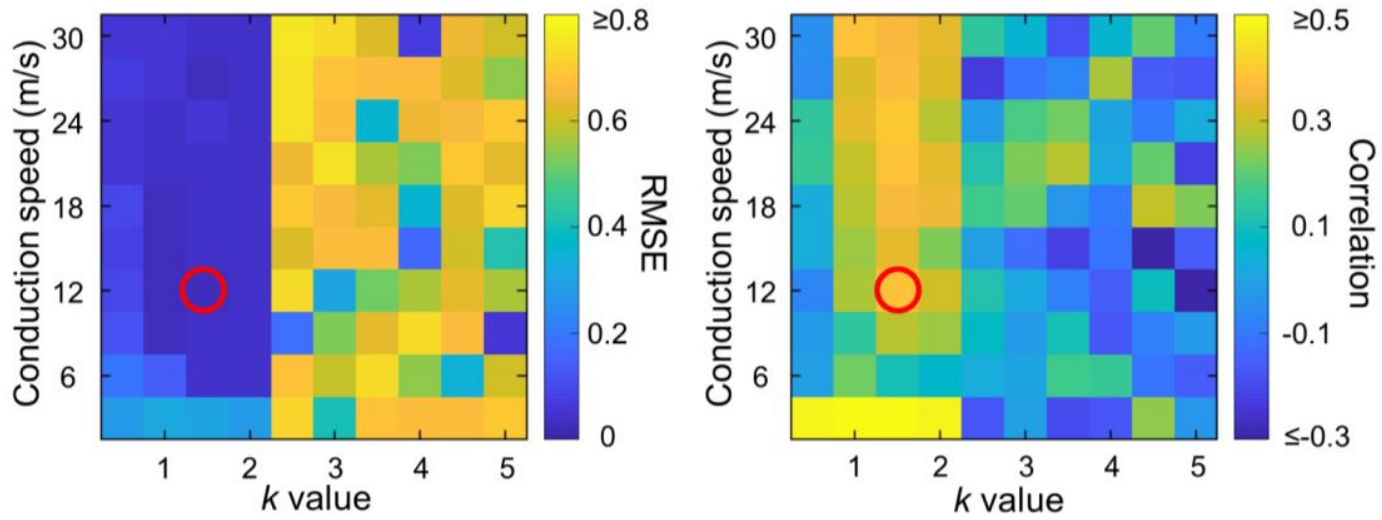

578 Figure 2. Grid search for optimal parameter values of each model. a. RMSE and

579 Correlation values for a range of $k$ values, for Wilson-Cowan zero delays model b. RMSE and

580 Correlation values for every combination of a range of $k$-values and a range of delay values,

581 for Wilson-Cowan constant delays model c. RMSE and Correlation values for every

582 combination of a range of $k$-values and range of conduction speed values, for Wilson-Cowan

583 distance-dependent delays model. $k$ is the scalar multiplier over the structural connectome. 


\subsection{Model comparison}

588 We compared the Wilson-Cowan zero delays, Wilson-Cowan constant delays and Wilson-

589 Cowan distance-dependent delays models. To do this, we compared the correspondences

590 between their respective model-generated networks of phase-synchronization, and the testing

591 set MEG-derived network of phase-synchronization. We quantified correspondence with

592 RMSE and Correlation between upper triangular elements of the model-generated matrices

593 from 20 simulations of each model and the testing set MEG-derived matrix. We used

594 independent samples $t$-tests to compare performances between models and Cohen's $d$ to

595 measure effect sizes. RMSE values from the constant delays model (mean $=0.041)$ are higher

596 than RMSE values from both the zero delays (mean $=0.039)\left(\right.$ two-tailed $p=1.1 \times 10^{-8}$,

597 Cohen's $d=2.3$ ) and distance-dependent delays models (mean $=0.039)$ (two-tailed $p=7.4 \times$

$59810^{-5}$, Cohen's $d=1.4$ ) (Figure 3, left panel). In contrast, there is no difference between RMSE

599 values from the zero delays and distance-dependent delays models (two-tailed $p>0.001$,

600 Cohen's $d=0.37$ ). Similarly, Correlation values from the constant delays model (mean =

601 0.37) are lower than Correlation values from the zero delays (mean $=0.47)$ (two-tailed $p=$

$6024.7 \times 10^{-10}$, Cohen's $d=2.62$ ) and distance-dependent delays models (mean = 0.46) (two-

603 tailed $p=1.6 \times 10^{-10}$, Cohen's $d=2.74$ ) (Figure 3, right panel), while there is no difference in

604 Correlation values from the zero delays and distance-dependent delays models (two-tailed $p>$

6050.001 , Cohen's $d=0.12$ ). These results suggest that the presence of delays in the Wilson-

606 Cowan distance-dependent delays model does not improve the correspondence between

607 model-generated and MEG-derived networks, compared to the correspondence obtained with

608 the Wilson-Cowan zero delays model. Hence, we chose the more parsimonious model, i.e.

609 the Wilson-Cowan zero delays model, for further investigation.

610 
612

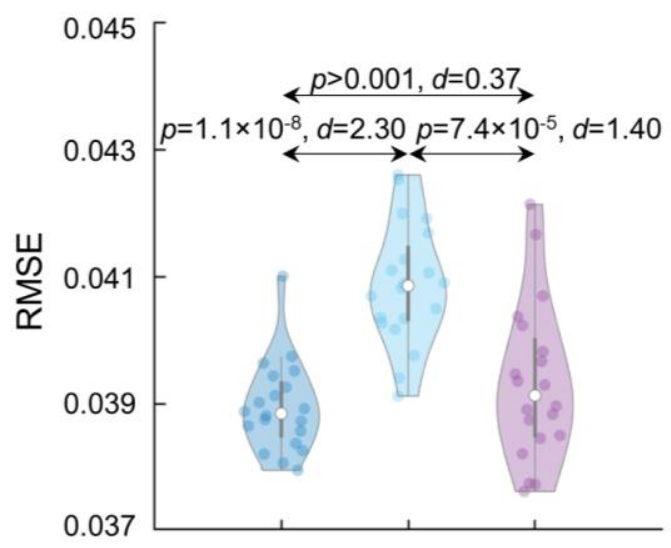

613

614

615

616

617

618

619

620

621

622

623

624

625

626

627

628

629

630

631

632

633

634 synchronization
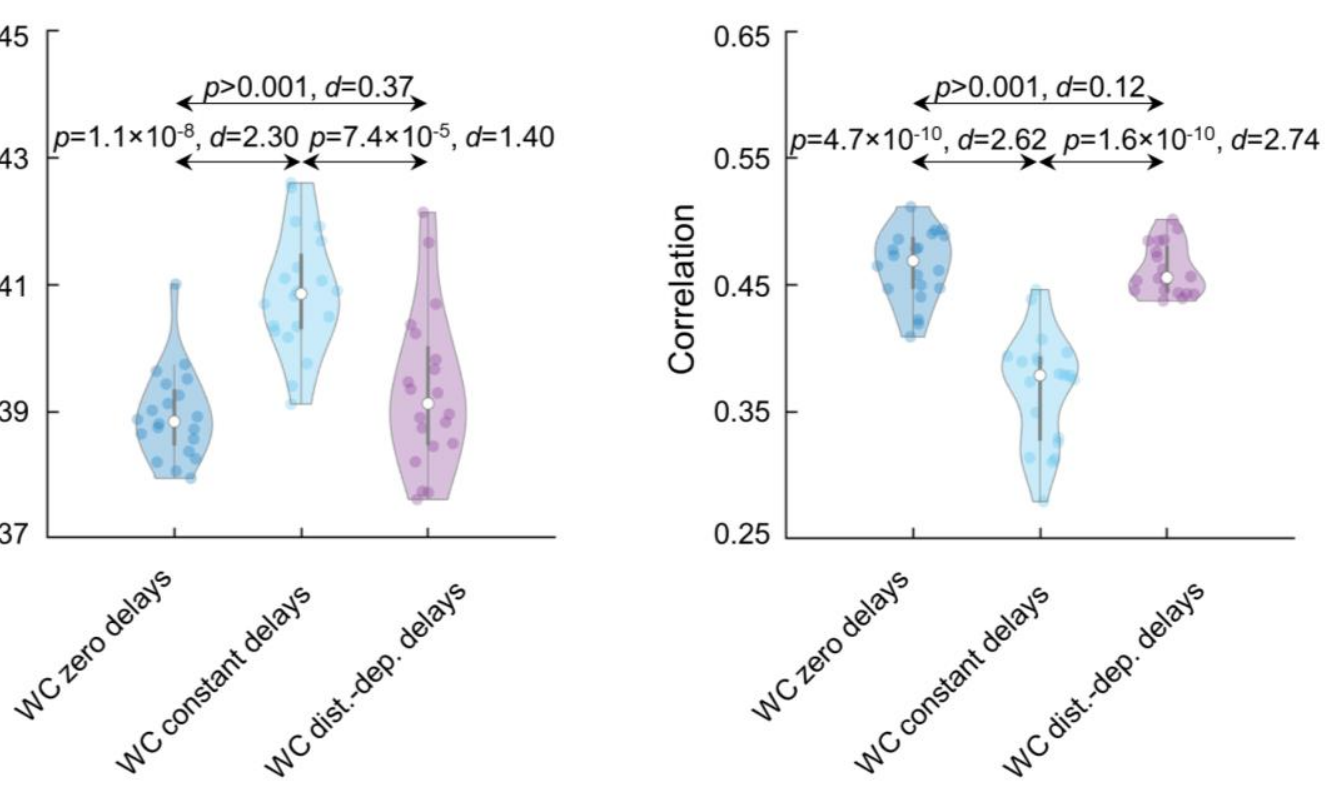

Figure 3. Including conduction delays does not improve model performance Violin plots of RMSE values for the Wilson-Cowan zero delays (mean $=0.039$ ), constant delays (mean = 0.041) and distance-dependent delays models (mean $=0.039)$ (left panel). Violin plots of Correlation values for the Wilson-Cowan zero delays $($ mean $=0.47)$, constant delays $($ mean $=$ 0.37 ) and distance-dependent delays models (mean =0.46) (right panel).

3.3 Close correspondence between model-generated and MEG-derived network of phase-

We observed that the source-reconstructed dynamics of the Wilson-Cowan zero delays model exhibited intermittent dynamics (Figure 4a), also observed in MEG resting-state data (Shriki et al. (2013)). Further, the frequency spectrum of the dynamics displayed peaks in delta (1 - 4 $\mathrm{Hz})$, alpha $(8-12 \mathrm{~Hz})$ and beta $(12-30 \mathrm{~Hz})$ frequency bands (Figure $4 \mathrm{~b})$, which have also been observed in MEG resting-state data (Mahjoory et al. (2020), Lopes da Silva (2013)).

We compared the levels of phase-synchronization within the source-reconstructed dynamics of the Wilson-Cowan zero delays model to those observed in source-reconstructed MEG restingstate data. To do this, we compared the mean of the Kuramoto order parameter (Breakspear et al. (2010)) of the source-reconstructed model dynamics and source-reconstructed MEG restingstate data, across participants in the held-out testing set. We observed mean of the Kuramoto order parameter of the model dynamics (Figure 4a) (mean $=8 \times 10^{-2}$, standard deviation $=9 \times$ $10^{-3}$ ) to be close to the mean of the Kuramoto order parameter of the source-reconstructed MEG resting-state data $\left(\right.$ mean $=8 \times 10^{-2}$, standard deviation $\left.=1 \times 10^{-2}\right)$. Hence, the source- 
reconstructed dynamics of the Wilson-Cowan zero delays model display similar levels of phase-synchronization as those observed in source-reconstructed MEG resting-state.

a.

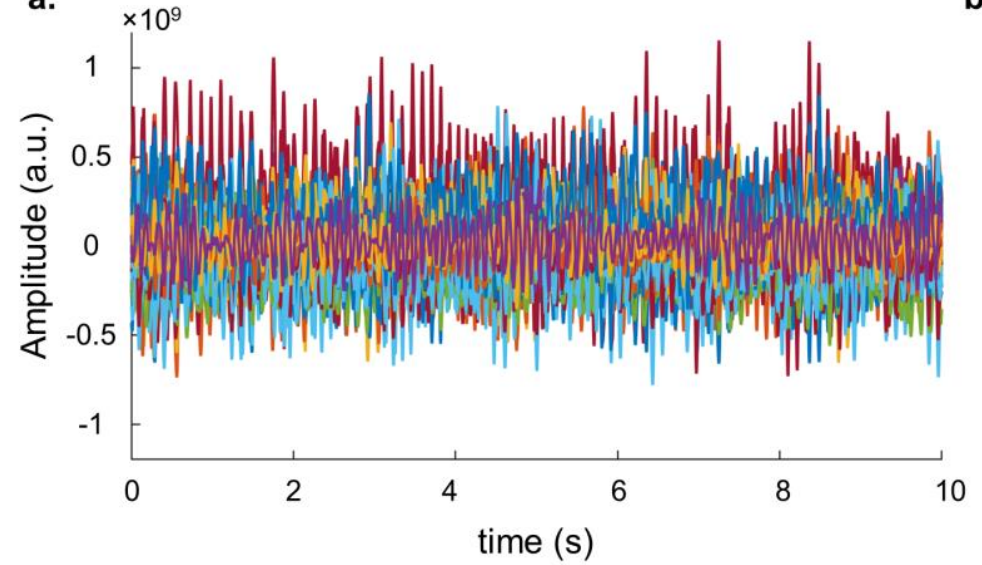

b.

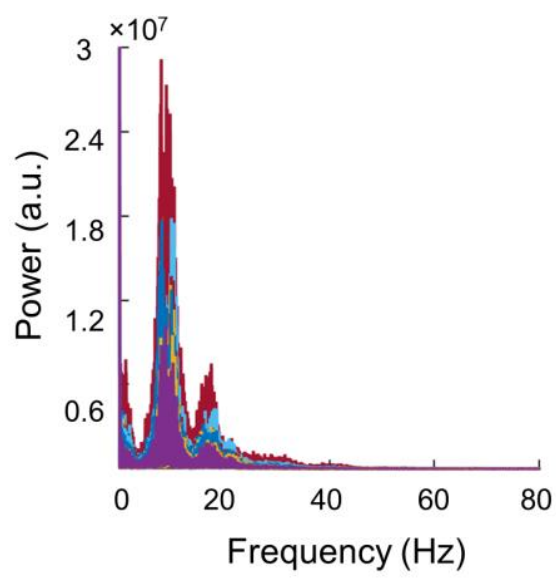

Figure 4. Intermittent dynamics and peaks in the frequency spectrum of dynamics of Wilson-Cowan zero delays model are similar to those observed in MEG resting-state. a. 10 -second time course of model dynamics from all brain regions for example subject $b$. Frequency spectrum of model dynamics for 10-second segment of model dynamics from all brain regions, for example subject. Different colours indicate time course/frequency spectrum of different brain regions. Same colour in panels $a, b$ belongs to the same brain region.

We next assessed the correspondence between the mean model-generated and MEG-derived networks of phase-synchronization. The mean model-generated network was the average of 20 model-generated networks, each estimated from simulated model data from different initial conditions. We compared the mean model-generated network of phase-synchronization to the testing set MEG-derived network. Specifically, we compared the distributions of mean modelgenerated and MEG-derived phase-synchronization values, and compared the RMSE and Correlation between the mean model-generated and MEG-derived matrices against their corresponding permutation-based null distributions. The distributions of mean modelgenerated and MEG-derived phase-synchronization values are similar, with central tendency $($ median $=0.09)$ and dispersion (median absolute deviation $=0.02)$ of mean model-generated network values close to central tendency (median $=0.07$ ) and dispersion (median absolute deviation $=0.02$ ) of MEG-derived network values (Figure 5a). RMSE $=0.04$ between mean model-generated and MEG-derived matrices is lower than chance $(z=-29.9$, one-tailed $p=6.7$ $\times 10^{-197}$ ), and Correlation $=0.49$ between mean model-generated and MEG-derived networks is higher than chance $(z=25.4$, one-tailed $p=0$ ) (Figure $5 b, c)$. Further, most top 5 percentile 
662 Taken together, the results demonstrate a close correspondence between the mean model663 generated and MEG-derived networks of phase-synchronization.

a.

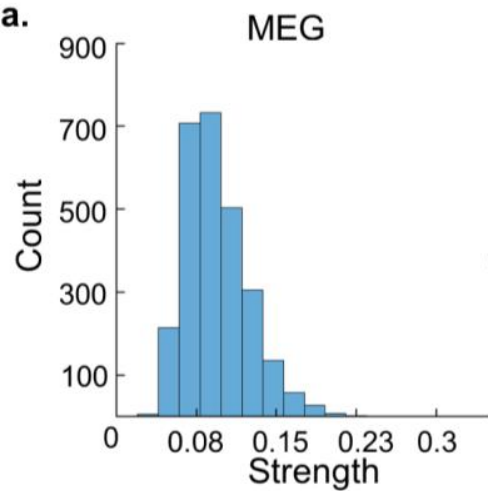

c.

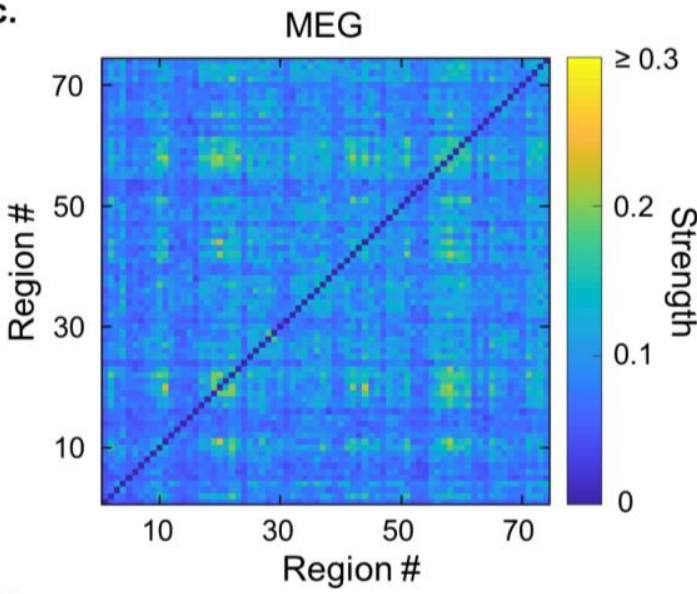

d.

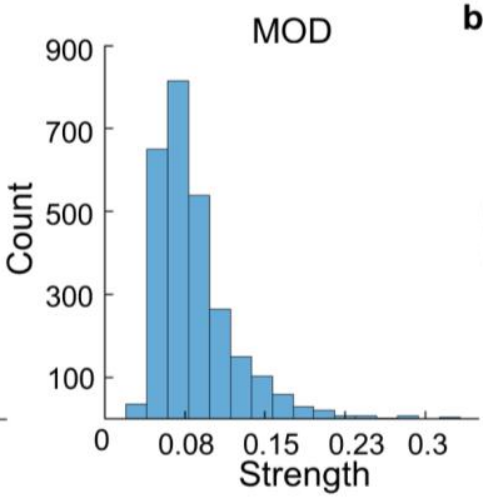

b.

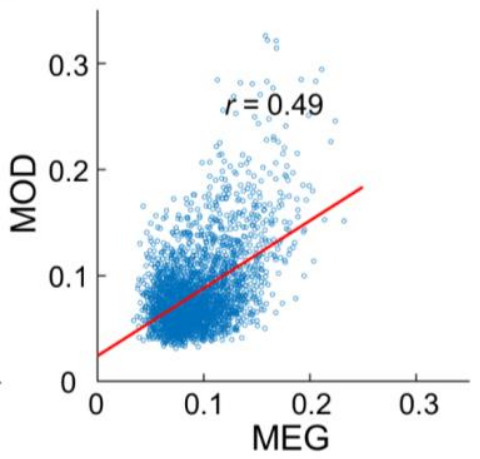

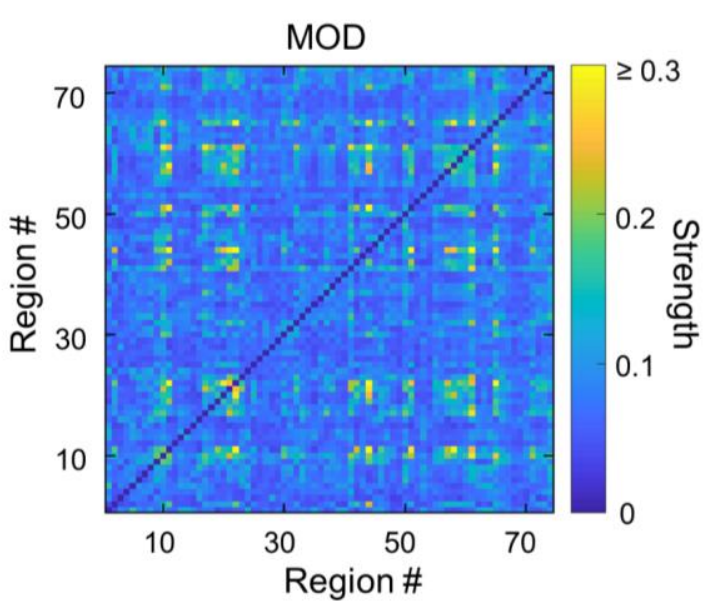
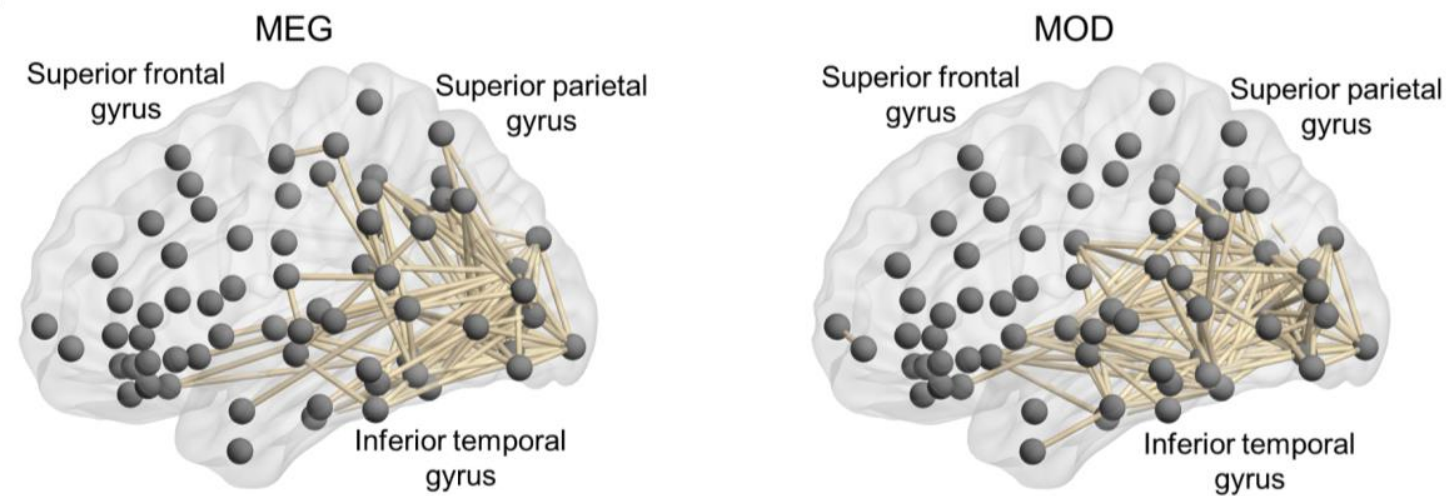

664 gyrus

665 Figure 5. Close correspondence between mean model-generated network and MEGderived network of phase-synchronization a. Histogram of connection strengths for mean model-generated and MEG-derived networks of phase-synchronization b. Scatter plot of connection strengths for MEG-derived network of phase-synchronization against mean modelgenerated network of phase-synchronization c. Matrices of connection strengths between every pair of brain regions for mean model-generated and MEG-derived network of phase- 
671

672

673

674

675

676

677

678

679

680

681

682

683

684

685

686

687

688

689

690

691

692

693

694

695

696

697

698

699

700

701

702

703

704

synchronization. d. Brain network visualisation of top 5 percentile strongest connections for mean model-generated and MEG-derived networks of phase-synchronization. MOD = model. Brain networks were visualised with BrainNet Viewer (http://www.nitrc.org/projects/bnv/) (Xia et al. (2013)).

We assessed the sensitivity of the Wilson-Cowan zero delays model, to changes in the values of model parameters. To do this, we performed a sensitivity analysis wherein we estimated change in model performance to small changes in the values of model parameters. We varied the value of each non-zero parameter from $-10 \%$ to $10 \%$ of its original value, while keeping all other parameters at their original value. The RMSE and Correlation from simulating these jittered models were $z$-scored against the 20 RMSE and Correlation values from simulating the original model, to estimate the change in model performance. We found the model performance to be robust to changes in values of $k$ and $w_{i i}$ parameters $(z$ values $<3)$, i.e. the scalar multiplier over the structural connectome and weight of self-inhibitory connections of Wilson-Cowan oscillators respectively. However, the model performance is sensitive to changes in the values of all other model parameters ( $z$ values $>3$ ) (Figure S1). Hence, we observe that the WilsonCowan zero delays model is sensitive to changes in the values of its model parameters.

We have shared the mean model-generated network and networks from each of 20 simulations of the Wilson-Cowan zero delays model, through an open dataset (Williams et al. (2021b)).

\subsection{Determining features of each model component contributing to model performance}

The Wilson-Cowan zero delays model represents the hypothesis that the Wilson-Cowan oscillatory dynamics interact with the pattern of structural connections to produce networks of phase-synchronization corresponding to MEG-derived networks of phase-synchronization. Hence, it postulates that both the pattern of structural connections and Wilson-Cowan oscillatory dynamics contribute to the observed correspondence.

We tested the hypothesis that the pattern of structural connections contributes to the correspondence between model-generated and MEG-derived networks. To do this, we compared correspondence obtained with the original model against correspondence obtained with null models containing degree-preserved randomised versions of the structural connectome, all other aspects of the null model being identical to the original model. The structural connectome from each of the null models had the same number of connections to 
each brain region as the original structural connectome, but the pattern of connections between regions was different from that of the original structural connectome. We used RMSE and Correlation between the model-generated and testing set MEG-derived matrices to quantify correspondence. Mean RMSE with the original model of 0.04 was lower $(z=-3.7$, one-tailed $\left.p=1 \times 10^{-4}\right)$ and mean Correlation of 0.47 was higher $(z=8.4$, one-tailed $p=0)$ than the set of RMSE and Correlation values yielded by null models containing degree-preserved randomised versions of the original structural connectome (Figure 6a). These results confirm that the pattern of connections in the structural connectome contributes to the observed correspondence between model-generated and MEG-derived networks, over and above the number of connections, i.e. degree, to each brain region.

Given the contribution of the pattern of connections of the structural connectome to the observed correspondence between model-generated and MEG-derived networks, we investigated if the model-generated network is merely a recapitulation of the structural connectome. To do this, we compared Correlation between the model-generated matrix and the testing set MEG-derived matrix, against Correlations between 100 bootstrapped versions of the structural connectome matrix and the testing set MEG-derived matrix. Mean Correlation of 0.47 between the model-generated and MEG-derived matrices is higher $(z=181.5$, one-tailed $p=0$ ) than Correlations between bootstrapped versions of the structural connectome matrix and the MEG-derived matrix (Figure S2). These results demonstrate that the model does not merely recapitulate the structural connectome at the level of its dynamics, but that it operates on the structural connectome in a non-trivial way to produce the observed MEG-derived matrix of phase-synchronization.

We tested the hypothesis that the Wilson-Cowan oscillatory dynamics contributes to the comparison between model-generated and MEG-derived networks of phase-synchronization. To do this, we compared correspondence obtained with the original model against correspondence obtained with null models wherein the dynamics of each brain region were described by random dynamics interacting through the structural connectome used in the original model. The mean and range of random dynamics were matched to those of the WilsonCowan oscillators. For the random node dynamics model also, we estimated the model parameter $k$, the scalar multiplier over the structural connectome, with the same procedure as for the Wilson-Cowan zero delays model (Section 2.6). We used RMSE and Correlation between the model-generated and testing set MEG-derived matrices to quantify 
correspondence. Note that Correlation is insensitive to scale of the compared phasesynchronization strengths, hence it would yield high values for similar patterns of modelgenerated and MEG-derived phase-synchronization even if the random node dynamics model produced weaker phase-synchronization strengths than those observed in the MEG-derived networks. Just as for the Wilson-Cowan zero delays model, the optimal value of $k$ for the random node dynamics model was identified as 2. Mean RMSE with the original model of 0.04 was lower $(z=-40.8$, one-tailed $p=0)$ and mean Correlation of 0.47 was higher $(z=4.9$, onetailed $p=5 \times 10^{-7}$ ) than the set of RMSE and Correlation values yielded by null models containing random node dynamics instead of Wilson-Cowan oscillatory dynamics (Figure 6b). These results confirm that the Wilson-Cowan oscillatory dynamics contributes to the observed correspondence between model-generated and MEG-derived networks, over and above dynamics with the same mean and range.

a.

Original v/s random connectome model

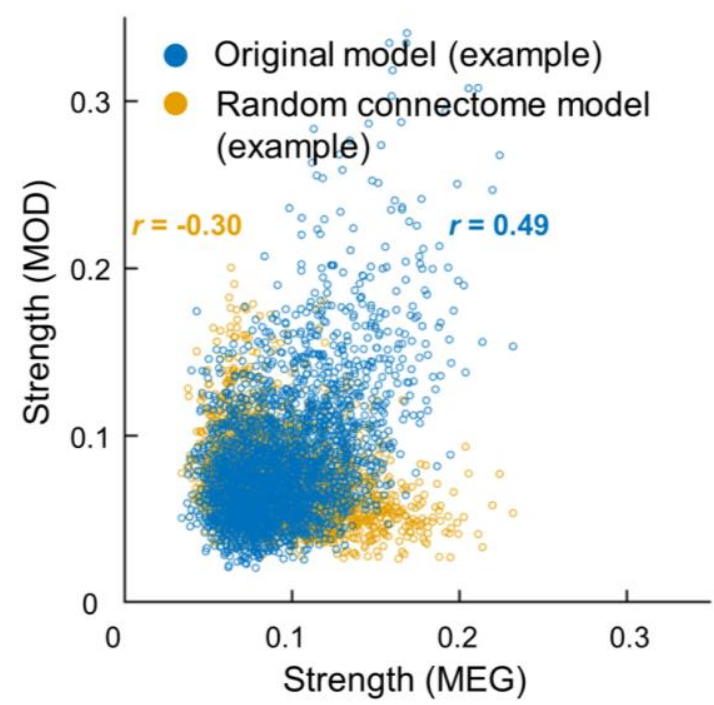

b. Original $\mathrm{v} / \mathrm{s}$ random node dynamics model

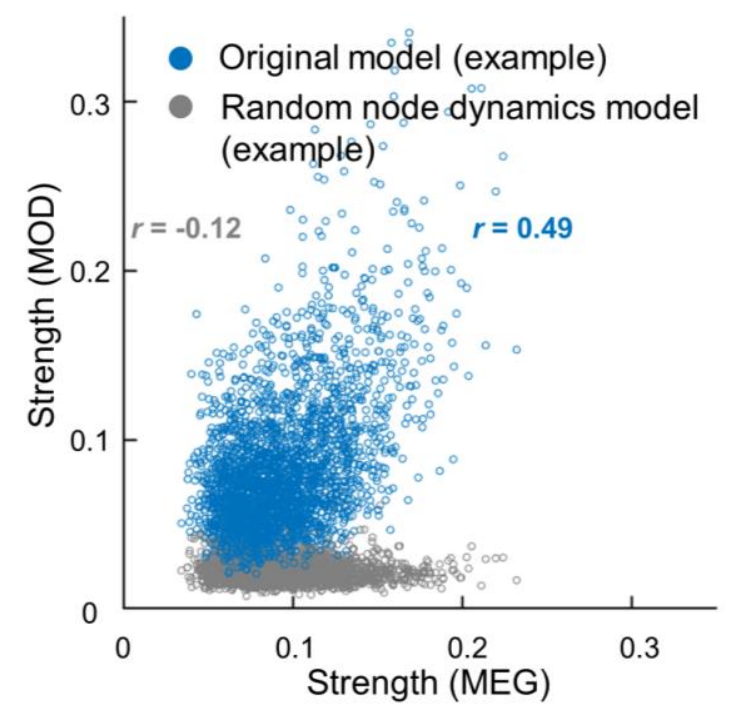

Figure 6. The pattern of connections in the structural connectome and dynamics of Wilson-Cowan oscillators, contribute to correspondence between model-generated and MEG-derived networks of phase-synchronization a. Scatter plot of connection strengths from MEG-derived network and example model-generated network (blue) and of connection strengths from MEG-derived network and example null model-generated network, wherein structural connectome is randomised (yellow). b. Scatter plot of connection strengths from MEG-derived network and example model-generated network (blue) and scatter plot of connection strengths from MEG-derived network and null model-generated network, wherein node dynamics are randomised (dark gray). MOD = model. 
Given the contribution of Wilson-Cowan oscillator dynamics to the correspondence between model-generated and MEG-derived networks, we investigated if the mere presence of oscillatory rather than random node dynamics would produce the observed correspondence. To do this, we compared correspondence obtained with the Wilson-Cowan zero delays model against correspondence obtained with an equivalent Kuramoto oscillator model, which also produces oscillatory dynamics. No statistically significant difference in the correspondences with the Wilson-Cowan zero delays model and Kuramoto oscillator model would imply the mere presence of oscillatory node dynamics produces a correspondence between modelgenerated and MEG-derived networks. We quantified correspondence using the RMSE and Correlation between the respective model-generated and MEG-derived matrices of phasesynchronization. We identified $k=0.5$ as the optimal value of the scalar multiplier over the structural connectome for the Kuramoto oscillator model. Mean RMSE of 0.04 with the Wilson-zero delays model was lower than mean RMSE of 0.53 with the Kuramoto oscillator model (two-tailed $p=8.4 \times 10^{-59}$ ). Similarly, mean Correlation of 0.47 with the Wilson-Cowan zero delays model is higher than mean Correlation of 0.13 with the Kuramoto oscillator model (two-tailed $p=2.2 \times 10^{-37}$ ) (Figure S3). These results demonstrate the mere presence of oscillatory node dynamics does not produce the observed correspondence between modelgenerated and MEG-derived networks. Rather, it is the oscillatory dynamics of the WilsonCowan oscillators resulting from the interaction between excitatory and inhibitory neuronal populations, which produces the observed correspondence.

\subsection{Robustness of results}

784 Finally, we investigated the robustness of the obtained correspondence between modelgenerated and MEG-derived networks, to choices made for the simulations and analyses. To do this, we compared correspondence obtained with different solvers and with different relative tolerance values for the model simulations. In addition, we determined if changing the modeled hemisphere from left to right would qualitatively change the correspondence between the model-generated and MEG-derived networks. We quantified correspondence by the RMSE and Correlation between the model-generated and MEG-derived matrices of phasesynchronization. We used the ODE45 solver in the original simulations, but RMSE and Correlation values are not different when ODE23 and ODE113 solvers were used (two-tailed $p>0.001$ for each) (Figure 7a). Similarly, we used relative tolerance of $10^{-3}$ for the original simulations, which yielded RMSE and Correlation values neither different to those obtained with lower relative tolerance values of $10^{-4}$ and $10^{-5}$ nor a higher relative tolerance of $10^{-2}$ (two- 
tailed $p>0.001$ for each) (Figure 7b). Changing the modeled hemisphere from left to right also yielded a moderate correspondence between the model-generated and MEG-derived network $($ Correlation $=0.33)$ (Figure S4). Hence, the correspondence between modelgenerated and MEG-derived networks of phase-synchronization, were robust to choices made 800 for the simulations and analyses.
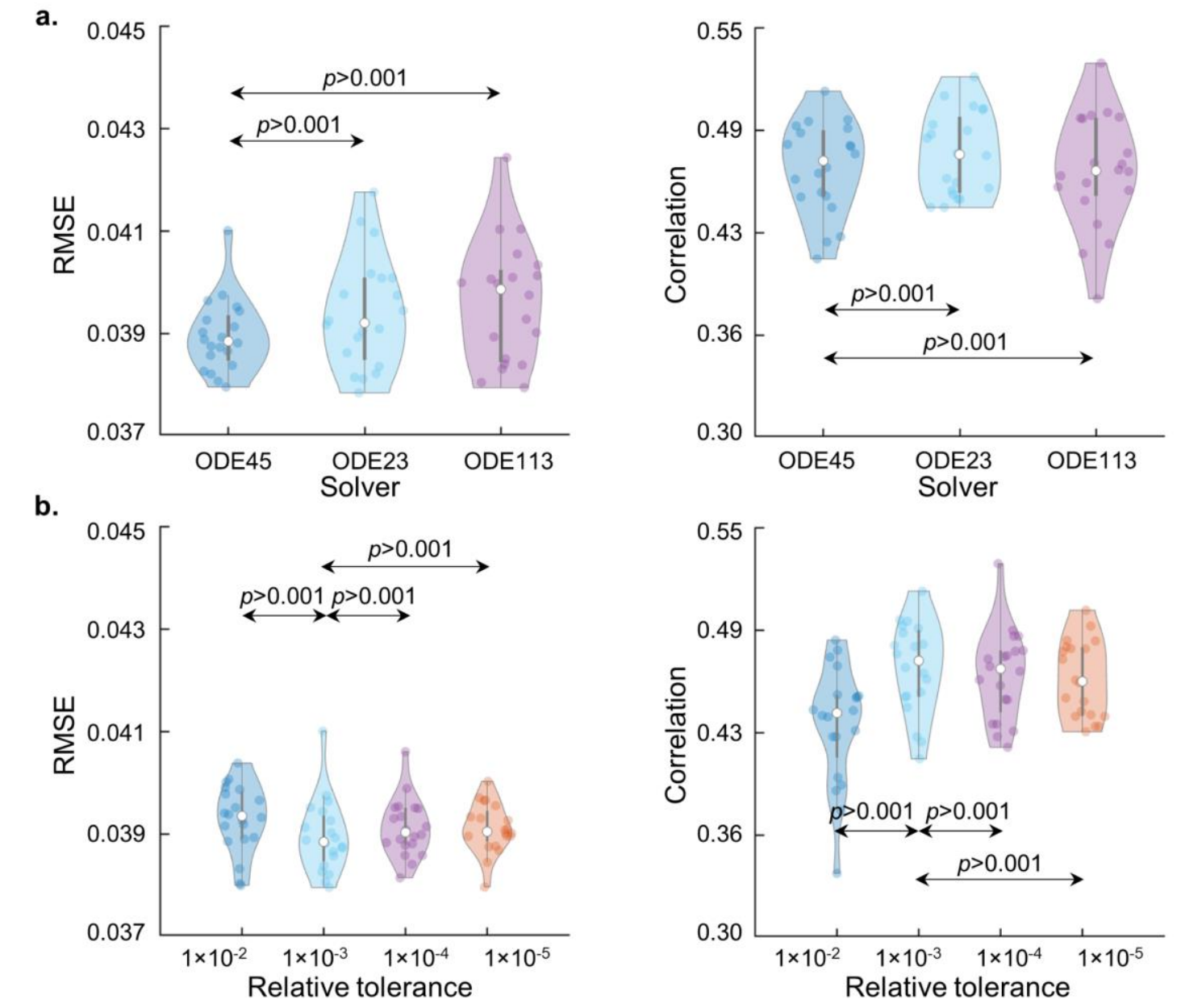

802 Figure 7. Results are robust to changes in solver and relative tolerance of solution a.

803 Boxplot of RMSE and Correlation with different solvers. Solver used in the original model was 804 ODE45. b. Boxplot of RMSE and Correlation at different relative tolerances of solution. 805 Relative tolerance used in the original model was $10^{-3}$.

\section{Discussion}

807 Large-scale networks of phase-synchronization are widely observed with EEG or MEG during 808 rest and task. BNMs are used to produce model-generated networks corresponding to MEG809 derived networks of phase-synchronization, but the respective roles of conduction delays, the structural connectome and dynamics of models of individual brain regions, in obtaining this 
811 correspondence remain unknown. In this paper, we investigated the roles of conduction delays,

812 the structural connectome and dynamics of models of individual regions, in producing 813 networks of phase-synchronization corresponding to those observed in MEG resting-state. We

814 found no evidence that including conduction delays improves the correspondence between the

815 model-generated and MEG-derived networks of phase-synchronization. Further, we

816 demonstrated that both the pattern of structural connections and Wilson-Cowan oscillatory

817 dynamics contribute to obtaining the observed correspondence between model-generated and

818 MEG-derived networks of phase-synchronization.

819

820 The topological organisation of the structural connectome, i.e., its pattern of connections, 821 contributes to the observed correspondence between the model-generated and MEG-derived

822 network of phase-synchronization. Previous studies have used the structural connectome within

823 BNMs to produce other phenomena, such as networks matching experimentally observed

824 fMRI-derived networks (Deco et al. (2009)), MEG-derived networks of amplitude correlation

825 (Tewarie et al. (2019)) and spatial distribution of oscillation amplitudes observed in MEG resting-state (Raj et al. (2019)). Studies have also used the structural connectome in models to

827 produce networks matching MEG-derived networks of phase-synchronization, but these have 828 been via abstract statistical or oscillator models (Finger et al. (2016)), graph theory measures 829 (Wodeyar \& Srinivasan (2019)) or formal series expansions (Meier et al. (2016)). A single 830 study used the structural connectome with a biologically plausible model to produce networks of phase-synchronization matching those observed in the MEG resting-state (Abeysuriya et al. (2018)). Our study advances findings from these studies in the following ways: 1.) We used comparison against null models to identify the specific feature of the structural connectome, i.e., its topological organisation, rather than lower-level properties such as the degree of each brain region, contributing to the observed correspondence between model-generated and MEGderived networks of phase-synchronization, and 2.) We demonstrated that the correspondence between the model-generated and MEG-derived networks of phase-synchronization is not explained merely by the correspondence between the structural connectome and the MEGderived network of phase-synchronization. Consistent with computational modelling on the relationship between the structural connectome and networks of phase-synchronization

841 (Forrester et al. (2020)), these results suggest a significant but nontrivial relationship between

842 the structural connectome and the model-generated network of phase-synchronization. 
844 The dynamics of Wilson-Cowan oscillators contribute to the observed correspondence between

845 model-generated and MEG-derived networks of phase-synchronization. Previous modelling

846 studies have studied the role of the structural connectome in producing observed functional

847 networks, but the role of dynamics of brain regions in producing these networks has not

848 received much attention. A recent computational modelling study (Forrester et al. (2020))

849 demonstrated the influence of node dynamics on the relationship between the structural 850 connectome and model-generated networks of phase-synchronization. The study demonstrated that oscillatory node dynamics are accompanied by a nontrivial relationship between the structural connectome and model-generated networks of phase-synchronization. Our study advances previous work in the following ways: 1.) While previous work has related oscillatory node dynamics to model-generated networks, this is the first work demonstrating the importance of oscillatory node dynamics in producing networks of phase-synchronization corresponding to those observed in experimental MEG resting-state. 2.) We illustrate the importance of specifically Wilson-Cowan oscillatory dynamics, by demonstrating that the Wilson-Cowan zero delays model produces networks with closer correspondence to MEGderived networks of phase-synchronization than does an equivalent model of Kuramoto oscillators. This result is consistent with computational modelling demonstrating the influence of both amplitude and phase dynamics, as produced by Wilson-Cowan oscillators, on estimates of phase-synchronization (Daffertshofer \& van Wijk (2011)). Kuramoto oscillators only yield descriptions of phase dynamics. Hence, the oscillatory dynamics resulting from the interaction between excitatory and inhibitory neuronal populations, as described by Wilson-Cowan oscillators, contributes to producing networks of phase-synchronization corresponding to those observed in MEG resting-state.

We find no evidence that including conduction delays improves the correspondence between model-generated and MEG-derived networks of phase-synchronization. Modelling studies investigating the role of delays in producing networks resembling fMRI-derived functional networks or MEG-derived networks of amplitude correlation have yielded differing results. Results of some studies suggest the importance of delays in producing fMRI-derived networks (Ghosh et al. (2008), Deco et al. (2009)) or MEG-derived networks of amplitude correlation (Cabral et al. (2014), Nakagawa et al. (2014)), while other modelling studies not including delays also produce networks resembling fMRI-derived (Honey et al. (2007)) or MEG-derived networks of amplitude correlation (Deco et al. (2017)). The role of conduction delays in producing networks resembling MEG-derived networks of phase-synchronization has not 
878

879

880

881

882

883

884

885

886

887

888

889

890

891

892

893

894

895

896

897

898

899

900

901

902

903

904

905

906

907

908

909

910

911

received much attention. A recent modelling study (Abeysuriya et al. (2018)) reported that, in the special case of a high mean activity level (mean activity $=0.3$ ) of Wilson-Cowan oscillators imposed by synaptic plasticity mechanisms, conduction delays were not necessary to producing networks resembling MEG-derived networks of phase-synchronization. In our study, we advance previous work by demonstrating that, also in the more general case of no specific mean target activity imposed by synaptic plasticity mechanisms, a close correspondence between model-generated and MEG-derived networks of phase-synchronization can be achieved.

The absence of evidence in our study, for the role of delays in producing MEG-derived networks of phase-synchronization, might be either due to errors in the estimates of conduction delay or due to conduction delays not being mechanistically important in producing MEGderived networks of phase-synchronization. If the latter were true, one would expect the Wilson-Cowan constant delays and Wilson-Cowan distance-dependent delays model to produce networks corresponding equally to the MEG-derived network of phasesynchronization. However, the Wilson-Cowan distance-dependent delays model produces networks correspondingly more closely to the MEG-derived network, than networks produced by the Wilson-Cowan constant delays model. In fact, the Wilson-Cowan distance-dependent delays model produces networks corresponding as closely to the MEG-derived network, as networks produced by the Wilson-Cowan zero delays model. Hence, we propose that improved accuracy in estimating conduction delays, by accounting for axonal diameter and myelination (Nakagawa et al. (2014), Waxman \& Swadlow (1977)) in addition to distance, could yield improved correspondence between networks produced by the Wilson-Cowan distancedependent delays model and the MEG-derived network of phase-synchronization. Specialised diffusion MRI sequences could yield estimates of myelination and axonal diameter (Drobnjak et al. (2016), Whittall et al. (1997)), but these estimates remain elusive.

The Wilson-Cowan zero delays model compares well to previously proposed BNMs producing networks of phase-synchronization observed in the MEG resting-state. A strength of a recently proposed BNM (Abeysuriya et al. (2018)) with distance-dependent conduction delays and inhibitory synaptic plasticity, was that it used the same set of parameter values to simultaneously produce a correspondence to both MEG-derived networks of amplitude correlation and MEG-derived networks of phase-synchronization. However, two notable aspects of the Wilson-Cowan zero delays model we propose are 1.) its parsimonious nature in not including conduction delays or synaptic plasticity mechanisms, while still producing 
912

913

914

915

916

917

918

919

920

921

922

923

924

925

926

927

928

929

930

931

932

933

934 Future work could extend the model to include empirically observed spatial gradients in 935

936

937

938

939

940

941

942

networks corresponding closely $(\rho=0.49)$ to MEG-derived networks of phase-synchronization and 2.) its strengths of phase-synchronization between regions were of the same order of magnitude and closer to those observed in the MEG-derived network (RMSE $=0.04)$ compared to previously proposed BNMs. However, a direct comparison between our model and those used in previous studies is difficult due to differences in the brain parcellation atlases used, number of hemispheres considered, measures of phase-synchronization used, as well as processing details of MEG and model-generated data.

A limitation of the Wilson-Cowan zero delays model we propose is that while it does produce networks corresponding to MEG-derived networks of phase-synchronization, it does not simultaneously produce networks of amplitude correlation nor produce spatial distribution of oscillation amplitudes corresponding to those observed in MEG resting-state (results not shown). However, we did find these phenomena were produced by the model operating at very low levels of structural coupling between regions, e.g., $k$ values of $10^{-3}$, where $k$ is the scalar multiplier across the structural connectome. This was outside the range of structural coupling strengths investigated in our study. Future studies will use likelihood-free inference methods to estimate parameters from wider parameter ranges (Lintusaari et al. (2017), Lintusaari et al. (2018), Gutmann \& Corander (2016), Cranmer et al. (2020)). Sensitivity analysis of our model revealed the model performance to be highly sensitive to values of model parameters. This could be due to the absence, in the model, of empirically observed homeostatic mechanisms to counteract the effect of changes in e.g., the mean activity level of the Wilson-Cowan oscillators. synaptic excitation (Wang (2020)), which in turn produce empirically observed spatial gradients in peak frequency (Hadida et al. (2019), Mahjoory et al. (2020)). The model could also be extended to include the effects of noise (Faisal et al. (2008)), which has been suggested to play a role in generating dynamics of resting-state data from other methodologies (Deco et al. (2009)). These models, fit to MEG resting-state data, could be locally perturbed to emulate task-related dynamics (Tiesinga et al. (2010)). Such virtual experiments could be used to develop, test and refine hypotheses on biophysical substrates underlying observed networks of phase-synchronization in MEG task data. 


\section{Conclusion}

944 In this study, we investigated the respective contributions of conduction delays, the structural

945 connectome and dynamics of models of individual brain regions, in producing model-generated

946 networks of phase-synchronization corresponding to those observed in MEG resting-state.

947 Based on our investigations, we report no evidence for the role of conduction delays in

948 producing networks corresponding to MEG-derived networks of phase-synchronization.

949 Further, we demonstrate the contribution of the topological organisation of the structural 950 connectome and the dynamics of Wilson-Cowan oscillators in producing model-generated 951 networks of phase-synchronization that correspond to those observed in MEG resting-state.

952 Future studies will extend these models and subject them to local perturbations to provide 953 insight on biophysical substrates underlying observed networks in rest and task conditions.

\section{Acknowledgements}

955 The authors gratefully acknowledge the Academy of Finland (NW: 321542, SK: 292334, 956 319264, MP: 253130, 256472, 281414, 296304, 266745, SP: 266402, 266745, 303933, 957 325404), Department of Science \& Technology (DST), India and Sigrid Juselius Foundation, 958 for providing funding for this project. The authors are particularly grateful to Prof. Sitabhra 959 Sinha and Prof. Mark Woolrich, for their invaluable comments and feedback, which 960 contributed to this work. The authors are also grateful to Alexander Aushev, Anton Mallasto, 961 Anirudh Jain and Diego Mesquita for comments on preliminary drafts of the manuscript.

\section{References}

1. Abeysuriya R., Hadida J., Sotiropoulus S., Jbabdi S., Becker R., Hunt B., Brookes M., Woolrich M. (2018) A biophysical model of dynamic balancing of excitation and inhibition in fast oscillatory large-scale networks. PLoS Comput Biol, 14(2): e1006007

2. Beckmann C., DeLuca M., Devlin J., Smith S. (2005) Investigations into resting-state connectivity using independent component analysis. Philos Trans $R$ Soc Lond B Biol Sci, 360(1457): 1001-1013

3. Binzegger T., Douglas R., Martin K. (2004) A quantitative map of the circuit of the cat primary visual cortex. J Neurosci, 24(39): 8441-8453 
4. Bogacki P., Shampine L. (1996) An efficient Runge-Kutta (4,5) pair. Comput Math with Appl, 32(6): 15-28

5. Breakspear M., Heitmann S., Daffertshofer, A. (2010) Generative models of cortical oscillations: neurobiological implications of the Kuramoto model. Front Hum Neurosci, 4, 190

6. Brookes M., Woolrich M., Barnes G. (2012) Measuring functional connectivity in MEG: a multivariate approach insensitive to linear source leakage. NeuroImage, 63(2): 910-920

7. Cabral J., Luckhoo H., Woolrich M., Joensson M., Mohseni H., Baker A., Kringelbach M., Deco G. (2014) Exploring mechanisms of spontaneous functional connectivity in MEG: how delayed network interactions lead to structured amplitude envelopes of band-pass filtered oscillations. NeuroImage, 90: 423-435.

8. Cohen J. (1988) Statistical Power Analysis for the Behavioural Sciences. New York, NY: Routledge Academic

9. Cohen J., D'Esposito M. (2016) The segregation and integration of distinct brain networks and their relationship to cognition. J Neurosci, 36(48): 12083-12094

10. Colclough G., Brookes M., Smith S., Woolrich M. (2015) A symmetric multivariate leakage correction for MEG connectomes. NeuroImage, 117: 439-448

11. Cole M., Reynolds J., Power J., Repovs G., Anticevic A., Braver T. (2013) Multi-task connectivity reveals flexible hubs for adaptive task control. Nat Neurosci, 16(9): 13481355

12. Cowan J., Neuman J., van Drongelen W. (2016) Wilson-Cowan equations for neocortical dynamics. J Math Neurosci 6, 1

13. Cranmer K., Brehmer J., Louppe G. (2020) The frontier of simulation-based inference. Proc Natl Acad Sci USA, 117(48): 30055-30062

14. da Silva F. (2013) EEG and MEG: Relevance to Neuroscience. Neuron, 80(5): 11121128

15. Daffertshofer A., van Wijk B. (2011) On the influence of amplitude on the connectivity between phases. Front NeuroInform. 5:6

16. Damoiseaux J., Rombouts S., Barkhof F., Scheltens P., Stam C., Smith S., Beckmann C. (2006) Consistent resting-state networks across healthy subjects. Proc Natl Acad Sci USA, 103(37): 13848-13853 
17. Deco G., Cabral J., Woolrich M., Stevner A., Van Hartevelt T., Kringelbach M. (2017) Single or multiple frequency generators in on-going brain activity: a mechanistic whole-brain model of empirical MEG data. NeuroImage, 152: 538-550

18. Deco G., Jirsa V., McIntosh A., Sporns O., Kötter R. (2009) Key role of coupling, delay and noise in resting brain fluctuations. Proc Natl Acad Sci USA, 106(25): 10302-10307

19. Deco G., Tononi G., Boly M., Kringelbach M. (2015) Rethinking segregation and integration: contributions of whole-brain modelling. Nat Rev Neurosci, 16(7): 430-439

20. Destrieux C., Fischl B., Dale A., Halgren E. (2010) Automatic parcellation of human cortical gyri and sulci using standard anatomical nomenclature. NeuroImage, 53(1): 115

21. Douglas R. Martin K., Whitteridge D. (1989) A canonical microcircuit for neocortex. Neural Comput, 1(4): 480-488

22. Drobnjak I., Zhang H., Ianus A., Kaden E., Alexander D. (2016) PGSE, OGSE and sensitivity to axon diameter in diffusion MRI: insight from a simulation study. Magn Reson Med, 75(2): 688-700

23. Faisal A., Selen L., Wolpert D. (2008) Noise in the nervous system. Nat Rev Neurosci, 9(4): 292-303

24. Finger H., Bönstrup M., Cheng B., Messé A., Hilgetag C., Thomalla G., Gerloff C., König P. (2016) Modeling of large-scale functional brain networks based on structural connectivity from DTI: comparison with EEG derived phase coupling networks and evaluation of alternative methods along the modeling path. PLoS Comput Biol, 12(8): e1005025

25. Forrester M., Crofts J., Sotiropoulos S., Coombes S., O’Dea R. (2020) The role of node dynamics in shaping emergent functional connectivity patterns in the brain. Netw Neurosci, 4(2): 467-483

26. Fries P. (2005) A mechanism for cognitive dynamics: neuronal communication through neuronal coherence. Trends Cogn Sci, 9(10): 474-480.

27. Ghosh A., Rho Y., McIntosh A., Kötter R., Jirsa V. (2008) Cortical network dynamics with time delays reveals functional connectivity in the resting brain. Cogn Neurodyn, 2(2): $115-120$

28. Gutmann M., Coriander J. (2016) Bayesian optimization for likelihood-free inference of simulator-based statistical models. J Mach Learn Res, 17: 1-47

29. Hadida J., Sotiropoulos S., Abeysuriya R., Woolrich M., Jbabdi S. (2018) Bayesian optimisation of large-scale biophysical networks. NeuroImage 174: 219-236 
30. Hadida J., Sotiropoulos S., Jbabdi S., Woolrich M. (2019) Capturing MEG spectral dynamics with non-uniform biophysical networks. Poster presented at: 25th Annual Meeting of the Organization for Human Brain Mapping (OHBM) 2019, June 9-13 2019, Rome, Italy

31. Hämäläinen M., Ilmoniemi R. (1994) Interpreting fields of the brain: minimum-norm estimates. Med Biol Eng Comput 32: 35-42

32. Heitmann S., Auburn M., Breakspear M. (2018) The Brain Dynamics Toolbox for MATLAB. Neurocomputing, 315: 82-88

33. Hipp J., Hawelleck D., Corbetta M., Siegel M, Engel A. (2012) Large-scale cortical correlation structure of spontaneous oscillatory activity. Nat Neurosci, 15(6): 884-890

34. Hirvonen J., Monto S., Wang S., Palva M., Palva S. (2018) Dynamic large-scale network synchronization from perception to action. Netw Neurosci, 2(4): 442-463

35. Hlinka J., Coombes S. (2012) Using computational models to relate structural and functional brain connectivity. Eur J Neurosci, 36(2): 2137-2145

36. Honey C., Kötter R., Breakspear M., Sporns O. (2007) Network structure of the cerebral cortex shapes functional connectivity on multiple time scales. Proc Natl Acad Sci USA, 104(24): 10240-10245

37. Jansen B., Rit V. (1995) Electroencephalogram and visual evoked potential generation in a mathematical model of coupled cortical columns. Biol Cybern, 73: 357-366

38. Kilpatrick Z. (2013) Wilson-Cowan model. In: Encyclopedia of Computational Neuroscience, edited by Jaeger D., Jung R. New York: Springer, 1-5

39. Kitzbichler M., Henson R., Smith M., Nathan P., Bullmore E. (2011) Cognitive effort drives workspace configuration of human brain functional networks. J Neurosci, 31(22): 8259-8270

40. Korhonen O., Palva S., Palva M. (2014) Sparse weightings for collapsing inverse solutions to cortical parcellations optimize M/EEG source reconstruction accuracy. $J$ Neurosci Methods, 226, 147-160

41. Kuramoto Y. (1984) Chemical Oscillations, Waves and Turbulence. Mineola, NY: Dover Publications.

42. Lintusaari J., Gutmann M., Dutta R., Kaski S., Corander J. (2017) Fundamentals and recent developments in Approximate Bayesian Computation. Syst Biol, 66(1): e66-e82

43. Lintusaari J., Vuollekoski H., Kangasraasio A., Skytén K., Jarvenpaa M., Marttinen P., Gutmann M., Vehtari A., Coriander J., Kaski S. (2018) ELFI: Engine for LikelihoodFree Inference. J Mach Learn Res, 19(16): 1-7 
44. Lobier M., Palva M., Palva S. (2018) High-alpha band synchronization across frontal, parietal and visual cortex mediates behavioural and neuronal effects of visuospatial attention. NeuroImage, 165: 222-237

45. Mahjoory K., Schoffelen J-M., Keitel A., Gross J. (2020) The frequency gradient of human resting-state oscillations follow cortical hierarchies. eLife 9:e53715

46. Marzetti L., Basti A., Chella F., D’Andrea A., Syrjälä J., Pizzella V. (2019) Brain functional connectivity through phase coupling of neuronal oscillations: a perspective from magnetoencephalography. Front. Neurosci, 13, 964

47. Maslov S., Sneppen K. (2002) Specificity and stability in topology of protein networks. Science, 296(5569): 910-913

48. Meier J., Tewarie P., Hillebrand A., Douw L., van Dijk B., Stufflebeam S., Van Miegham P. (2016) A mapping between structural and functional brain networks. Brain Connect, 6(4): 298-311

49. Nakagawa T., Woolrich M., Luckhoo H., Joensson M, Mohseni H., Kringelbach M., Jirsa V., Deco G. (2014) How delays matter in an oscillatory whole-brain spikingneuron network model for MEG alpha-rhythms at rest. NeuroImage, 87: 383-394.

50. Noori R., Park D., Griffiths J., Bells S., Frankland P., Mabbott D., Lefebvre J. (2020) Activity-dependent myelination: a glial mechanism of oscillatory self-organization in large-scale brain networks. Proc Natl Acad Sci USA, 117(24): 13227-13237

51. Oostenveld R., Fries P., Maris E., Schoffelen J-M. (2011) FieldTrip: Open source software for advanced analysis of MEG, EEG and invasive electrophysiological data. Comput Intell Neurosci 2011(1): 156869

52. Palva M., Monto S., Kulashekhar S., Palva S. (2010) Neuronal synchrony reveals working memory networks and predicts individual memory capacity. Proc Natl Acad Sci USA, 107(16): 7580-7585

53. Palva M., Palva S., Kaila K. (2005) Phase synchrony among neuronal oscillations in the human cortex. Journal of Neuroscience, 25 (15) 3962-3972

54. Palva M., Wang S., Palva S., Zhigalov A., Monto S., Brookes M., Schoffelen J., Jerbi K. (2018) Ghost interactions in MEG/EEG source space: A note of caution on interareal coupling measures. NeuroImage, 173: 632-643

55. Palva S., Palva M. (2012) Discovering oscillatory interaction networks with M/EEG: challenges and breakthroughs. Trends Cogn Sci, 16(4): 219-230 
56. Pfeiffer C., Andersen L., Lundqvist D., Hämäläinen M., Schneiderman J., Oostenveld R. (2018) Localizing on-scalp MEG sensors using an array of magnetic dipole coils. PLoS ONE 13(5): e0191111

57. Raj A., Cai C., Xie X., Palacios E., Owen J., Mukherjee P., Nagarajan S. (2020) Spectral graph theory of brain oscillations. Hum Brain Mapp, 41(11): 2980-2998

58. Shampine L., Thompson S. (2001) Solving DDEs in MATLAB. Appl Numer Math, 37(4): 441-458

59. Shriki O., Alstott J., Carver F., Holroyd T., Henson R., Smith M., Coppola R., Bullmore E., Plenz D. (2013) Neuronal avalanches in the resting MEG of the human brain. Journal Neurosci. 33(16): 7079-7090

60. Siebenhühner F., Wang S., Arnulfo G., Lampinen A., Nobili L., Palva M., Palva S. (2020) Genuine cross-frequency coupling networks in human resting-state electrophysiological recordings. PLoS Biol, 18(5): e3000685

61. Siegel M., Donner T., Engel A. Spectral fingerprints of large-scale neuronal interactions. Nat Rev Neurosci, 13(2): 121-134

62. Singer W. (1999) Neuronal synchrony: a versatile code for definition of relations? Neuron, 24(1): 49-65

63. Singh R., Menon S., Sinha S. (2016) Complex patterns arise through spontaneous symmetry breaking in dense homogeneous networks of neural oscillators. Sci Rep 6(1): $1-8$

64. Smith R., Tournier J., Calamante F., Connelly A. (2012) Anatomically-constrained tractography: improved diffusion MRI streamlines tractography through effective use of anatomical information. NeuroImage, 62(3): 1924-1938

65. Smith R., Tournier J., Calamante F., Connelly A. (2013) SIFT: Spherical-deconvolution informed filtering of tractograms. NeuroImage, 67: 298-312

66. Sreenivasan V., Menon S., Sinha S. (2017) Emergence of coupling-induced oscillations and broken symmetries in heterogenously driven nonlinear reaction networks. Sci Rep 7(1): $1-8$

67. Taulu S., Hari R. (2009) Removal of magnetoencephalographic artifacts with temporal signal-space separation: demonstration with single-trial auditory-evoked responses. Hum Brain Mapp, 30: 1524-1534

68. Tewarie P., Abeysuriya R., Byrne Á., O’Neill G., Sotiropoulos S., Brookes M., Coombes S. (2019) How do spatially distinct frequency specific MEG networks emerge 
from one underlying structural connectome? The role of structural eigenmodes. NeuroImage, 186: 211-220

69. Tiesinga P., Sejnowski T. (2010) Mechanisms for phase shifting in cortical networks and their role in communication through coherence. Front Hum Neurosci, 4, 196

70. Traub R., Jefferys J., Whittington M. (1997) Simulation of gamma rhythms in networks of interneurons and pyramidal cells. J Comput Neurosci 4(2): 141-150

71. Van Essen D., Smith S., Barch D., Behrens T., Yacoub E., Ugurbil K., WU-Minn HCP Consortium (2013) The WU-Minn Human Connectome Project: an overview. NeuroImage, 80: 62-79

72. Vinck M., Oostenveld R., Wingerden M., Battaglia F., Pennartz C. (2011) An improved index of phase-synchronization for electrophysiological data in the presence of volumeconduction, noise and sample-size bias. NeuroImage, 55(4): 1548-1565

73. Wang X-J. (2020) Macroscopic gradients of synaptic excitation and inhibition in the neocortex. Nat Rev Neurosci, 21: 169-178

74. Waxman S., Swadlow H. (1977) The conduction properties of axons in central white matter. Prog Neurobiol, 8(4): 297-324

75. Whittall K., Mackay A., Graeb D., Nugent R., Li D., Paty D. (1997) In vivo measurement of $\mathrm{T}_{2}$ distributions and water contents in normal human brain. Magn Reson Med, 37(1): 34-43

76. Williams (2021a, May 28). MATLAB implementations of networks of WilsonCowan oscillators with delayed interactions (Version 1). Zenodo. http://doi.org/10.5281/zenodo.4817655

77. Williams N., Toselli B., Siebenhühner F., Palva S., Arnulfo G., Kaski S. Palva M. (2021b), "Connectomes of phase-synchronization generated by Wilson-Cowan network model", Mendeley Data, V1, doi: 10.17632/bgs7w9z24h.1

78. Wilson H., Cowan J. (1972) Excitatory and inhibitory interactions in localized populations of model neurons. Biophys J, 12(1): 1-24

79. Wodeyar A., Srinivasan R. (2019) Contribution of structural connectivity to MEG functional connectivity. biorxiv, 785600

80. Woolrich M., Stephan K. (2013) Biophysical network models and the human connectome. NeuroImage, 80: 330-338.

81. Xia M., Wang J., He Y. (2013) BrainNet Viewer: A network visualization tool for human brain connectomics. PLoS ONE, 8: e68910 
82. Zhigalov A., Arnulfo G., Nobili L., Palva S., Palva M. (2015) Relationship of fast-andslow-timescale neuronal dynamics in human MEG and SEEG. J Neurosci, 35(13): $5385-5396$

83. Zhigalov A., Arnulfo G., Nobili L., Palva S., Palva M. (2017) Modular co-organization of functional connectivity and scale-free dynamics in the human brain. Netw Neurosci

1174 1(2):143-165

1175

1176

1177

1178

1179

1180

1181

1182 


\section{Supplementary figures}

1188

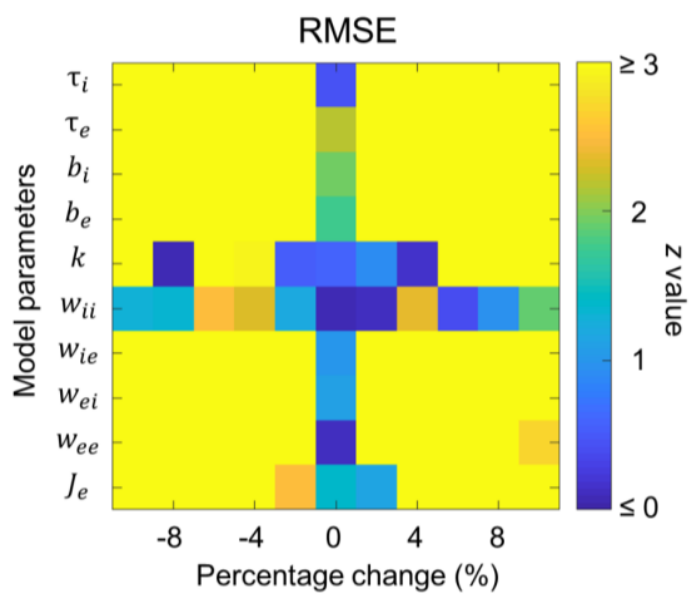

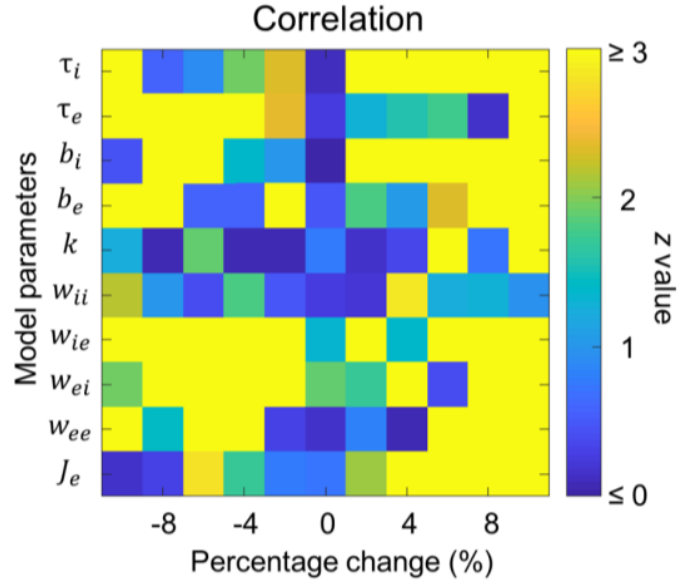

1189 Figure S1. Model performance is sensitive to values of model parameters Change in model performance, as measured by $z$-scored RMSE and $z$-scored Correlation, for percentage change in original value of each model parameter while keeping all other parameters at their original values. 


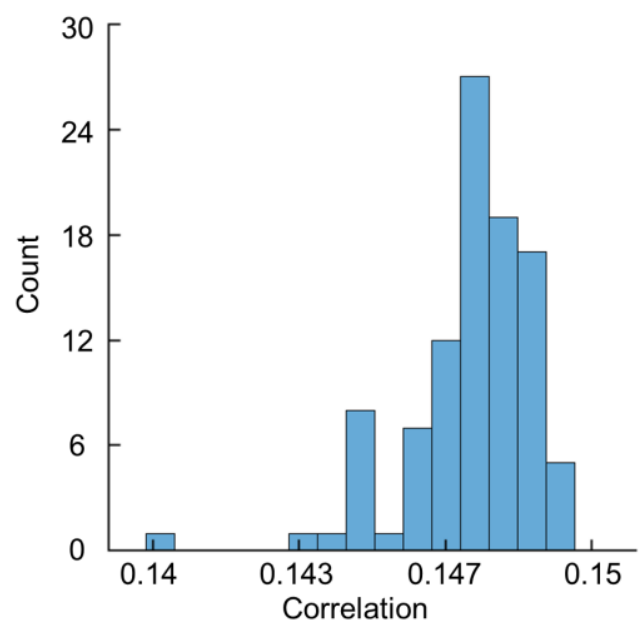
phase-synchronization Histogram of correlations between MEG-derived network of phase-

1203 synchronization and 100 bootstrapped versions of structural connectome.

1204

1205

1206

1207

1208

1209

1210

1211

1212

1213

1214

1215 

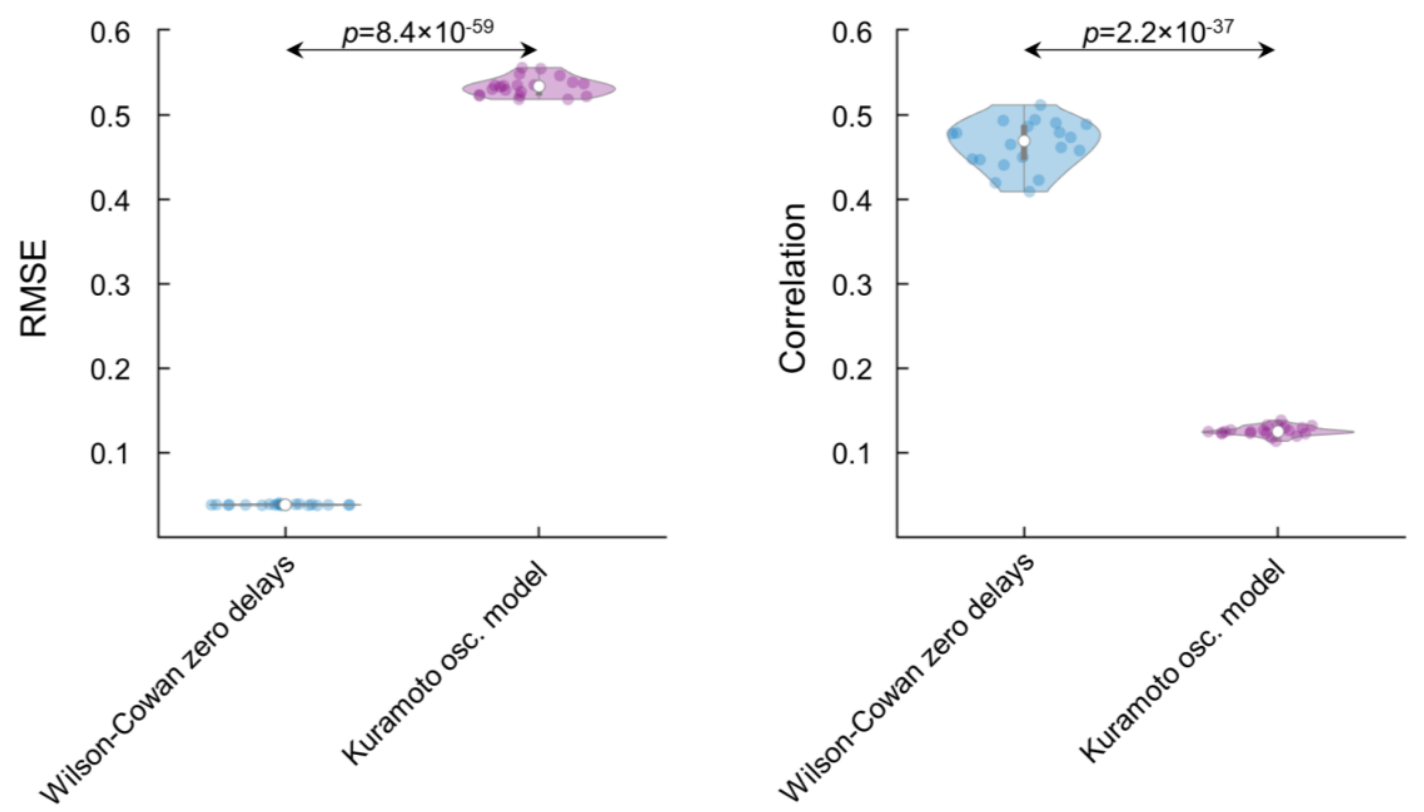

1217 Figure S3. Wilson-Cowan zero delays model outperforms Kuramoto oscillator model a.

1218 Violin plots of RMSE values for the Wilson-Cowan zero delays and Kuramoto oscillator 1219 models (left panel). Violin plots of Correlation values for the Wilson-Cowan zero delays and Kuramoto oscillator models (right panel).

1221

1222

1223

1224

1225

1226

1227

1228

1229

1230 
a.

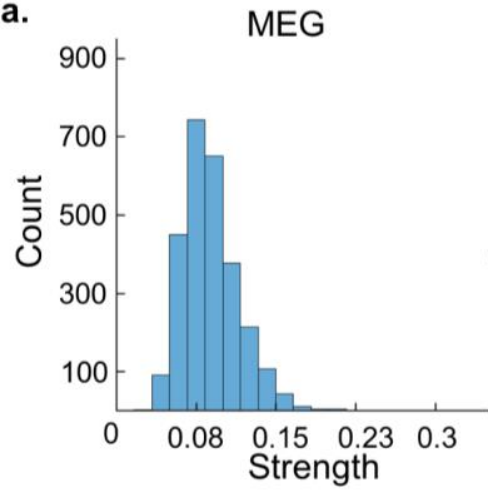

c.

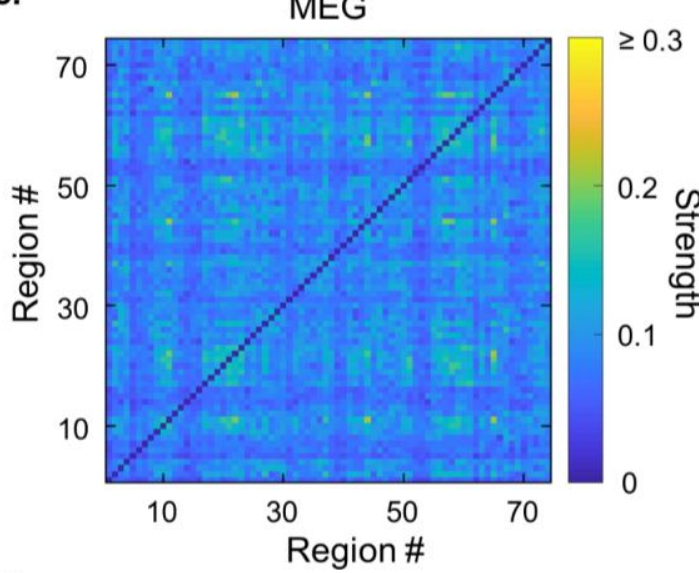

d.

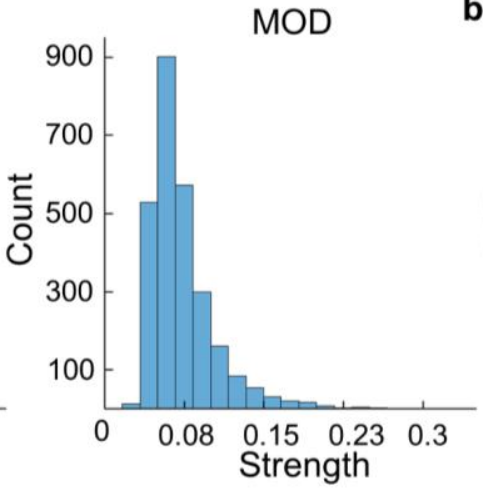

b.

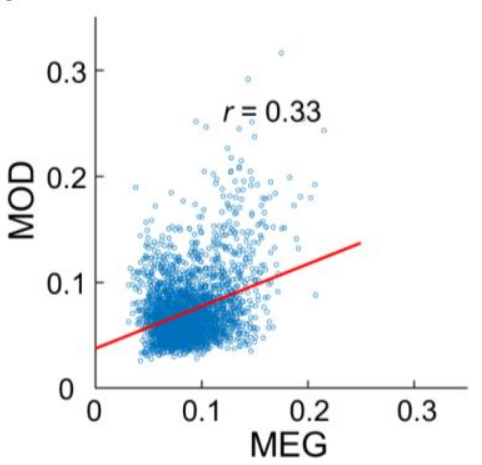

MEG

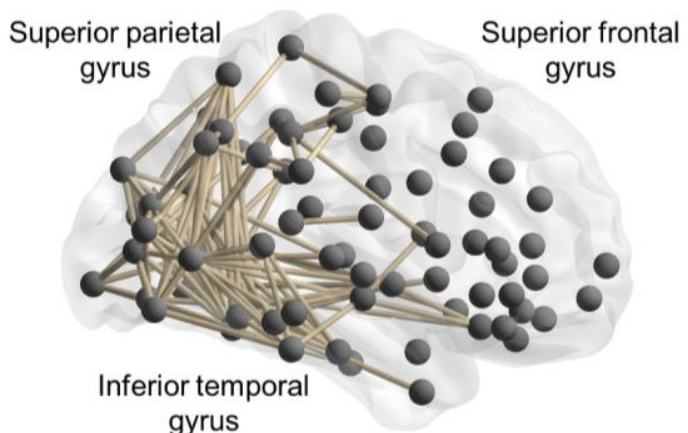

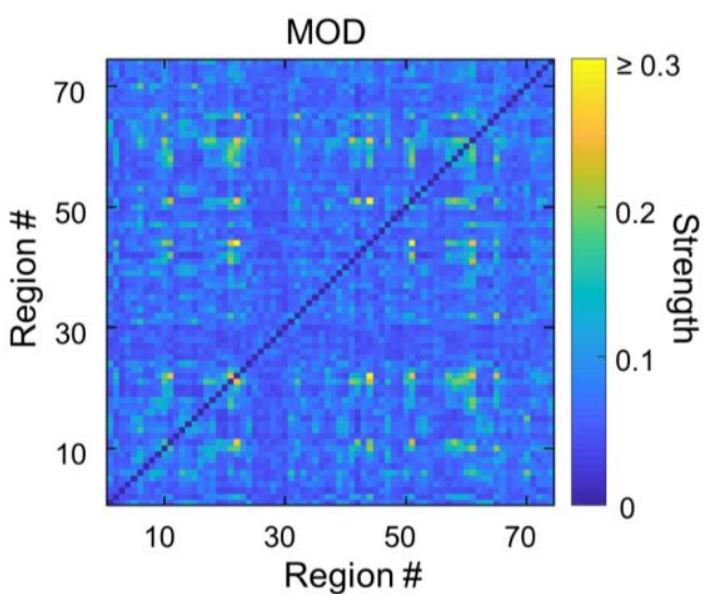

MOD

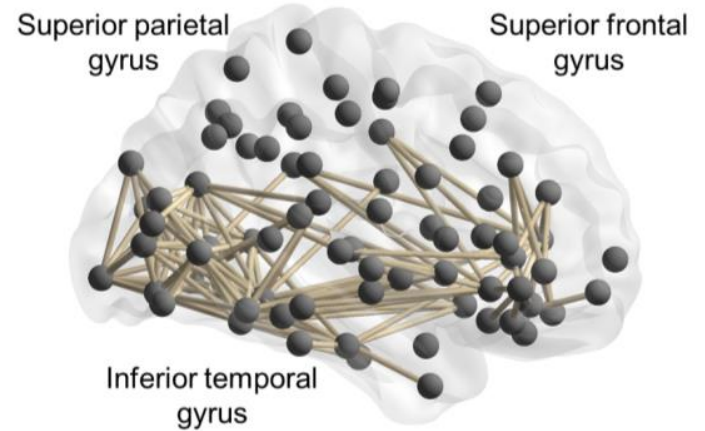

Figure S4. Moderate correspondence between mean model-generated network and rightstrengths for mean model-generated and right-hemispheric MEG-derived networks of phasesynchronization b. Scatter plot of connection strengths for right-hemispheric MEG-derived network of phase-synchronization against mean model-generated network of phasesynchronization c. Matrices of connection strengths between every pair of brain regions for mean model-generated and right-hemispheric MEG-derived network of phasesynchronization. d. Brain network visualisation of top 5 percentile strongest connections for mean model-generated and right-hemispheric MEG-derived networks of phase- 
bioRxiv preprint doi: https://doi.org/10.1101/2021.08.04.455014; this version posted August 5, 2021. The copyright holder for this preprint (which was not certified by peer review) is the author/funder, who has granted bioRxiv a license to display the preprint in perpetuity. It is made available under aCC-BY-NC-ND 4.0 International license.

1244 synchronization. MOD $=$ model. Brain networks were visualised with BrainNet Viewer 1245 (http://www.nitrc.org/projects/bnv/) (Xia et al. (2013)). 